\title{
Leptoquarks and matter unification: Flavor anomalies and the muon $g-2$
}

\author{
Pavel Fileviez Pérez $\oplus^{1,{ }^{*}}$ Clara Murgui $\oplus^{2, \dagger}$ and Alexis D. Plascencia $\oplus^{1, \$}$ \\ ${ }^{1}$ Physics Department and Center for Education and Research in Cosmology and Astrophysics (CERCA), \\ Case Western Reserve University, Cleveland, Ohio 44106, USA \\ ${ }^{2}$ Walter Burke Institute for Theoretical Physics, California Institute of Technology, \\ Pasadena, California 91125, USA
}

(Received 5 May 2021; revised 1 July 2021; accepted 4 August 2021; published 31 August 2021)

\begin{abstract}
We discuss the minimal theory for quark-lepton unification at the low scale. In this context, the quarks and leptons are unified in the same representations and neutrino masses are generated through the inverse seesaw mechanism. The properties of the leptoquarks predicted in this theory are discussed in detail, and we investigate the predictions for the leptonic and semileptonic decays of mesons. We study the possibility to explain the current value of $\mathcal{R}_{K}$ reported by the LHCb Collaboration and the value of the muon anomalous magnetic moment reported by the Muon $g-2$ experiment at Fermilab.
\end{abstract}

DOI: 10.1103/PhysRevD.104.035041

\section{INTRODUCTION}

The idea of quark-lepton unification by Pati and Salam [1] provides a simple and elegant approach to think about unification of matter and interactions in nature. The minimal theory of Pati-Salam is very predictive because it predicts, at the scale where matter unifies, that the masses of the charged leptons and down quarks are equal, and the masses for the up-quarks are equal to the Dirac masses for neutrinos. Moreover, the $\mathrm{SU}(4)_{C}$ gauge symmetry must be broken around the canonical seesaw scale, $10^{14} \mathrm{GeV}$, in order to achieve small neutrino masses, via the seesaw mechanism [2-5], in agreement with experiments.

The simplest quark-lepton unification theory that can be realized at the tera-electron-volt $(\mathrm{TeV})$ scale was proposed in Ref. [6]. This theory is based on the $\mathrm{SU}(4)_{C} \otimes$ $\mathrm{SU}(2)_{L} \otimes \mathrm{U}(1)_{R}$ gauge group, and in order to have a consistent theory for fermion masses at the low scale, neutrino masses are generated through the inverse seesaw mechanism $[7,8]$. This theory for quark-lepton unification predicts, among the new fields required for its consistency, the existence of mediators that interact simultaneously with both leptons and quarks. Particularly, this theory predicts a vector leptoquark, $X_{\mu} \sim(\mathbf{3}, \mathbf{1}, 2 / 3)_{\mathrm{SM}}$, and two scalar leptoquarks, $\Phi_{3} \sim(\overline{\mathbf{3}}, \mathbf{2},-1 / 6)_{\mathrm{SM}}$ and $\Phi_{4} \sim(\mathbf{3}, \mathbf{2}, 7 / 6)_{\mathrm{SM}}$,

\footnotetext{
pxf112@case.edu cmurgui@caltech.edu

*alexis.plascencia@case.edu
}

Published by the American Physical Society under the terms of the Creative Commons Attribution 4.0 International license. Further distribution of this work must maintain attribution to the author(s) and the published article's title, journal citation, and DOI. Funded by SCOAP ${ }^{3}$. which mediate exotic processes that could otherwise not be seen in the context of the Standard Model (SM). ${ }^{1}$ For a review about the phenomenology of leptoquarks see Ref. [9].

Recently, the LHCb Collaboration has reported results for the ratio $\mathcal{R}_{K}$ defined as

$$
\mathcal{R}_{K}=\frac{\operatorname{Br}\left(B^{+} \rightarrow K^{+} \mu^{+} \mu^{-}\right)}{\operatorname{Br}\left(B^{+} \rightarrow K^{+} e^{+} e^{-}\right)}
$$

which is predicted to be 1 in the SM. The measurement reported by $\mathrm{LHCb}[10]$ using data from Run 2 is

$$
\mathcal{R}_{K}^{\exp }\left(1.1<q^{2}<6.0 \mathrm{GeV}^{2}\right)=0.846_{-0.039-0.012}^{+0.042+0.013}
$$

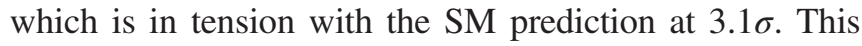
observable, together with $\mathcal{R}_{K^{*}}$ and the leptonic decays $\operatorname{Br}\left(B_{s} \rightarrow \ell^{+} \ell^{-}\right)$, where $\ell=e, \mu$, are usually classified as clean observables; in the former the hadronic and the long distance effects cancel almost exactly. These experimental results have motivated many studies in the particle physics community; see e.g., [11-35].

There are also new results reported by the Fermilab Muon $g-2$ experiment on the anomalous magnetic moment of the muon $a_{\mu}$ from their Run 1 [36]. The combined result with the one from the E821 experiment at BNL [37] deviates from the SM prediction ${ }^{2}$ by $4.2 \sigma$,

\footnotetext{
${ }^{1}$ We label with the subscript SM the quantum numbers of the corresponding field under the Standard Model gauge group $\mathrm{SU}(3)_{C} \otimes \mathrm{SU}(2)_{L} \otimes \mathrm{U}(1)_{Y}$.

${ }^{2}$ There are results from lattice QCD that are compatible with the experimental value [38].
} 


$$
\Delta a_{\mu}=a_{\mu}^{\exp }-a_{\mu}^{\mathrm{SM}}=(251 \pm 59) \times 10^{-11} .
$$

There have been different proposals of theories beyond the SM to explain this anomaly; see e.g., Refs. [39-54]. These results for $\mathcal{R}_{K}$ and $(g-2)_{\mu}$, which are naturally expected if new physics is around the multi-TeV scale, could help us find a new direction for physics beyond the Standard Model.

In this article, we investigate the possibility to explain the experimental value of $\mathcal{R}_{K}$ in two main scenarios. In the first scenario the scalar leptoquark $\Phi_{3} \sim(\overline{\mathbf{3}}, \mathbf{2},-1 / 6)_{\mathrm{SM}}$ gives the main contribution to the relevant meson decays, while in the second scenario the scalar leptoquark $\Phi_{4} \sim$ $(\mathbf{3}, 2,7 / 6)_{S M}$ plays the main role to explain the value of $\mathcal{R}_{K}$. In the second scenario, $\Phi_{4}$ couples mostly to electrons as required by constraints from lepton flavor violation. We also show that the component $\phi_{4}^{5 / 3}$ of $\Phi_{4}$, due to the enhancement by the factor $m_{t} / m_{\mu}$, can explain the reported value of the anomalous magnetic moment of the muon by the Muon $g-2$ Collaboration. Moreover, we show that the theory of minimal quark and lepton unification can address simultaneously the anomaly in $\mathcal{R}_{K^{(*)}}$ and the experimental value of $(g-2)_{\mu}$. In these scenarios, $\Phi_{3}$ and $\Phi_{4}$ explain the flavor anomalies while $\Phi_{4}$ addresses the $(g-2)_{\mu}$ through its couplings mainly to muons, so that the predictions are consistent with constraints from lepton flavor violation.

This article is organized as follows: in Sec. II we discuss the minimal theory for quark-lepton unification at the low scale, in Sec. III we investigate the predictions for meson decays and discuss the different leptoquark candidates to explain the experimental value of $\mathcal{R}_{K}$. In Sec. IV we discuss the possibility to explain the recent experimental results for the $g-2$ of the muon and demonstrate that the theory can address the anomalies in the clean observables involving $b \rightarrow s$ transitions and the muon $g-2$. Finally, in Sec. V we summarize our main findings.

\section{MINIMAL THEORY FOR QUARK-LEPTON UNIFICATION}

The minimal theory for quark-lepton unification that can describe physics at the TeV scale was proposed in Ref. [6]. This theory is based on the gauge symmetry,

$$
\mathcal{G}_{\mathrm{QL}}=\mathrm{SU}(4)_{C} \otimes \mathrm{SU}(2)_{L} \otimes \mathrm{U}(1)_{R},
$$

and the SM matter fields are unified as

$$
\begin{gathered}
F_{\mathrm{QL}}=\left(\begin{array}{ll}
u & \nu \\
d & e
\end{array}\right) \sim(\mathbf{4}, \mathbf{2}, 0), \\
F_{u}=\left(\begin{array}{ll}
u^{c} & \nu^{c}
\end{array}\right) \sim(\overline{\mathbf{4}}, \mathbf{1},-1 / 2), \\
\text { and } F_{d}=\left(\begin{array}{ll}
d^{c} & e^{c}
\end{array}\right) \sim(\overline{\mathbf{4}}, \mathbf{1}, 1 / 2) .
\end{gathered}
$$

Here all the SM fields and $\nu^{c}$ are in the left-handed representation, and the unification for quarks and leptons is for each SM family. This theory can be seen as a low energy limit of the Pati-Salam model based on $\mathrm{SU}(4)_{C} \otimes \mathrm{SU}(2)_{L} \otimes \mathrm{SU}(2)_{R}$, when the $\mathrm{SU}(2)_{R}$ symmetry is broken to $\mathrm{U}(1)_{R}$. One can also obtain the gauge symmetry $\mathcal{G}_{\mathrm{QL}}$ from a unified theory based on $\mathrm{SU}(6)$.

In this theory the gauge fields live in

$$
A_{\mu}=\left(\begin{array}{cc}
G_{\mu} & X_{\mu} / \sqrt{2} \\
X_{\mu}^{*} / \sqrt{2} & 0
\end{array}\right)+T_{4} B_{\mu}^{\prime} \sim(\mathbf{1 5}, \mathbf{1}, 0),
$$

where $G_{\mu} \sim(\mathbf{8}, \mathbf{1}, 0)_{\mathrm{SM}}$ are the gluons, $X_{\mu} \sim(\mathbf{3}, \mathbf{1}, 2 / 3)_{\mathrm{SM}}$ are vector leptoquarks, and $B_{\mu}^{\prime} \sim(\mathbf{1}, \mathbf{1}, 0)_{\mathrm{SM}}$. The Higgs sector is composed of three scalar representations:

$$
\begin{aligned}
H_{1}^{T} & =\left(\begin{array}{ll}
H^{+} & H^{0}
\end{array}\right) \sim(\mathbf{1}, \mathbf{2}, 1 / 2) \\
\chi & =\left(\begin{array}{ll}
\chi_{u} & \chi_{R}^{0}
\end{array}\right) \sim(\mathbf{4}, \mathbf{1}, 1 / 2), \quad \text { and } \\
\Phi & =\left(\begin{array}{cc}
\Phi_{8} & \Phi_{3} \\
\Phi_{4} & 0
\end{array}\right)+\sqrt{2} T_{4} H_{2} \sim(\mathbf{1 5}, \mathbf{2}, 1 / 2) .
\end{aligned}
$$

Here $H_{2} \sim(\mathbf{1}, \mathbf{2}, 1 / 2)_{\mathrm{SM}}$ is a second Higgs doublet, $\Phi_{8} \sim(\mathbf{8}, \mathbf{2}, 1 / 2)_{\mathrm{SM}}$, and the scalar leptoquarks $\Phi_{3} \sim$ $(\overline{\mathbf{3}}, \mathbf{2},-1 / 6)_{\mathrm{SM}}$ and $\Phi_{4} \sim(\mathbf{3}, \mathbf{2}, 7 / 6)_{\mathrm{SM}}$. The $T_{4}$ generator of $\mathrm{SU}(4)_{C}$ in the above equation is normalized as $T_{4}=\frac{1}{2 \sqrt{6}} \operatorname{diag}(1,1,1,-3)$. The $\mathcal{G}_{\mathrm{QL}}$ gauge group is spontaneously broken to the SM gauge group by the vacuum expectation value (VEV) of the scalar field $\left\langle\chi_{R}^{0}\right\rangle=v_{\chi} / \sqrt{2}$, which gives mass to the vector leptoquark $X_{\mu}$, defining the scale of matter unification.

The Yukawa interactions in this theory are given by

$$
\begin{aligned}
-\mathcal{L}_{\mathrm{QL}}^{Y}= & Y_{1} F_{\mathrm{QL}} F_{u} H_{1}+Y_{2} F_{\mathrm{QL}} F_{u} \Phi+Y_{3} H_{1}^{\dagger} F_{\mathrm{QL}} F_{d} \\
& +Y_{4} \Phi^{\dagger} F_{\mathrm{QL}} F_{d}+\text { H.c. },
\end{aligned}
$$

and the mass matrices for the SM fermions read as

$M_{U}=Y_{1} \frac{v_{1}}{\sqrt{2}}+\frac{1}{2 \sqrt{3}} Y_{2} \frac{v_{2}}{\sqrt{2}}, \quad M_{\nu}^{D}=Y_{1} \frac{v_{1}}{\sqrt{2}}-\frac{\sqrt{3}}{2} Y_{2} \frac{v_{2}}{\sqrt{2}}$,

$M_{D}=Y_{3} \frac{v_{1}}{\sqrt{2}}+\frac{1}{2 \sqrt{3}} Y_{4} \frac{v_{2}}{\sqrt{2}}, \quad M_{E}=Y_{3} \frac{v_{1}}{\sqrt{2}}-\frac{\sqrt{3}}{2} Y_{4} \frac{v_{2}}{\sqrt{2}}$.

Here the VEVs of the Higgs doublets are defined as $\left\langle H_{1}^{0}\right\rangle=$ $v_{1} / \sqrt{2}$ and $\left\langle H_{2}^{0}\right\rangle=v_{2} / \sqrt{2}$. Notice that without the scalar field $\Phi$ one cannot generate a consistent relation for charged fermion masses. Now, in order to generate small neutrino masses at the low scale one needs to go beyond the 
canonical seesaw mechanism. We can generate small Majorana masses for the light neutrinos if we add three new singlet left-handed fermionic fields $S \sim(\mathbf{1}, \mathbf{1}, 0)$ and use the following interaction terms [6], which emerge in the Lagrangian once the fermion singlets are included,

$$
-\mathcal{L}_{\mathrm{QL}}^{\nu}=Y_{5} F_{u} \chi S+\frac{1}{2} \mu S S+\text { H.c. }
$$

In this case the mass matrix for neutrinos in the basis $\left(\nu, \nu^{c}, S\right)$ reads as

$$
\left(\nu \nu^{c} S\right)\left(\begin{array}{ccc}
0 & M_{\nu}^{D} & 0 \\
\left(M_{\nu}^{D}\right)^{T} & 0 & M_{\chi}^{D} \\
0 & \left(M_{\chi}^{D}\right)^{T} & \mu
\end{array}\right)\left(\begin{array}{c}
\nu \\
\nu^{c} \\
S
\end{array}\right) .
$$

Here $M_{\nu}^{D}$ is given by Eq. (8) and $M_{\chi}^{D}=Y_{5} v_{\chi} / \sqrt{2}$. The light neutrino mass is given by

$$
m_{\nu} \approx \mu\left(M_{\nu}^{D}\right)^{2} /\left(M_{\chi}^{D}\right)^{2}
$$

if $M_{\chi}^{D} \gg M_{\nu}^{D} \gg \mu$ holds. Such a hierarchy is motivated by the different scales of the theory: $M_{\chi}^{D} \propto v_{\chi}$, which determines the scale of matter unification, $M_{\nu}^{D} \propto v_{1,2}$, which defines the electroweak scale, and $\mu$ is instead protected by a fermion symmetry, so that it is technically natural to assume it is small. Notice that neutrinos would be massless in the limit $\mu \rightarrow 0$, which is the usual relation in the inverse seesaw mechanism.

The vector leptoquarks, $X_{\mu} \sim(\mathbf{3}, \mathbf{1}, 2 / 3)_{\mathrm{SM}}$, have the following interactions:

$$
\mathcal{L} \supset \frac{g_{4}}{\sqrt{2}} X_{\mu}\left(\bar{Q}_{L} \gamma^{\mu} \ell_{L}+\bar{u}_{R} \gamma^{\mu} \nu_{R}+\bar{d}_{R} \gamma^{\mu} e_{R}\right)+\text { H.c. },
$$

where the gauge coupling $g_{4}$ is equal to the strong coupling constant evaluated at the quark-lepton unification scale and $\left(\nu_{R}\right)^{c}=\left(\nu^{c}\right)_{L}$. See Appendix A for details of the interactions in the physical basis.

The Yukawa interactions in Eq. (7), other than generating the mass of the fermions, contain new Yukawa interactions with respect to the Standard Model. Particularly, the predicted scalar leptoquarks, $\Phi_{3} \sim(\overline{\mathbf{3}}, \mathbf{2},-1 / 6)_{\mathrm{SM}}$ and $\Phi_{4} \sim(\mathbf{3}, 2,7 / 6)_{\mathrm{SM}}$, have the following interactions with quarks and leptons:

$$
\begin{aligned}
-\mathcal{L}_{\mathrm{QL}}^{Y} \supset & Y_{2} Q_{L} \Phi_{3}\left(\nu^{c}\right)_{L}+Y_{2} \ell_{L} \Phi_{4}\left(u^{c}\right)_{L}+Y_{4} \ell_{L} \Phi_{3}^{\dagger}\left(d^{c}\right)_{L} \\
& +Y_{4} Q_{L} \Phi_{4}^{\dagger}\left(e^{c}\right)_{L}+\text { H.c. }
\end{aligned}
$$

We note that neutrino masses can be small even when $Y_{2} \rightarrow 0$, due to the inverse seesaw mechanism, but the entries in $Y_{4}$ cannot be arbitrarily small because one needs a realistic relation between down quarks and charged lepton masses,

$$
Y_{4}=\sqrt{\frac{3}{2}} \frac{1}{v_{2}}\left(M_{D}-M_{E}\right)
$$

The scalar leptoquarks $\Phi_{3}$ and $\Phi_{4}$ can be written in $\mathrm{SU}(2)_{L}$ components as

$$
\Phi_{3}=\left(\begin{array}{c}
\phi_{3}^{1 / 3} \\
\phi_{3}^{-2 / 3}
\end{array}\right) \text { and } \Phi_{4}=\left(\begin{array}{c}
\phi_{4}^{5 / 3} \\
\phi_{4}^{2 / 3}
\end{array}\right),
$$

where the numbers in the superscript denote the electric charge. In Appendix A we present the interactions of the leptoquarks in the physical basis, where the fermions are mass eigenstates. For some phenomenological studies of this theory see, for example, Refs. [25,55].

\section{MESON DECAYS: $\mathcal{R}_{K}$ AND $\mathcal{R}_{K^{*}}$}

The theory predicts the existence of a vector leptoquark, $X_{\mu}$, and two scalar leptoquarks, $\Phi_{3}$ and $\Phi_{4}$, among other fields. The interactions of the $X_{\mu}$ leptoquark are determined by several unknown unitary mixing matrices; see Appendix A for details. Unfortunately, one cannot explain easily the values of $\mathcal{R}_{K}$ and satisfy the bounds from the experimental constraints on $K_{L} \rightarrow e^{ \pm} \mu^{\mp}$ when the mixing matrix is unitary. See the studies in Refs. [25,33] for details. Consequently, we focus this study on the scalar leptoquarks that the theory predicts.

The interactions of the scalar leptoquarks with the Standard Model fermions are needed to render the fermion masses realistic, and therefore cannot be assumed small. In particular, in Eqs. (8) and (9) one can see that $Y_{2}$ can be neglected, but $Y_{4}$ must be nonzero in order to have a consistent relation between the charged leptons and down quark masses. For simplicity, in this section we will study scenarios where we take the limit $Y_{2} \rightarrow 0$, and hence, the interactions of the scalar leptoquarks with the SM fermions are given by

$$
\begin{aligned}
-\mathcal{L}_{Y}= & Y_{4}^{a b}\left(\bar{d}_{R}^{b}\left(\phi_{3}^{1 / 3}\right)^{*} \nu_{L}^{a}+\bar{d}_{R}^{b}\left(\phi_{3}^{-2 / 3}\right)^{*} e_{L}^{a}+\bar{e}_{R}^{b}\left(\phi_{4}^{5 / 3}\right)^{*} u_{L}^{a}\right. \\
& \left.+\bar{e}_{R}^{b}\left(\phi_{4}^{2 / 3}\right)^{*} d_{L}^{a}\right)+ \text { H.c. }
\end{aligned}
$$

which in the basis where the fermions are mass eigenstates read

$$
\begin{aligned}
-\mathcal{L}_{Y}= & \bar{d}_{R}^{b} V_{4}^{a b}\left(\phi_{3}^{1 / 3}\right)^{*} \nu_{L}^{a} \\
& +\bar{d}_{R}^{b}\left(K_{3}^{*} V_{\mathrm{PMNS}}^{*} V_{4}\right)^{a b}\left(\phi_{3}^{-2 / 3}\right)^{*} e_{L}^{a}+\bar{e}_{R}^{b} V_{6}^{a b}\left(\phi_{4}^{5 / 3}\right)^{*} u_{L}^{a} \\
& +\bar{e}_{R}^{b}\left(K_{2} V_{\mathrm{CKM}}^{T} K_{1} V_{6}\right)^{a b}\left(\phi_{4}^{2 / 3}\right)^{*} d_{L}^{a}+\text { H.c. }
\end{aligned}
$$

To determine the parameters quantifying the leptoquark interaction with fermions, i.e., the corresponding coupling and the leptoquark mass, the predictions of the theory should be contrasted with experimental measurements. Strikingly, both scalar leptoquarks contribute to $b \rightarrow s$ 
transitions through their coupling between the charged leptons and down quarks, via the $\phi_{3}^{-2 / 3}$ and $\phi_{4}^{2 / 3}$ fields. Therefore, in light of recent deviations reported by the $\mathrm{LHCb}$ on such transitions, we should ask the theory to accommodate the experimental results, being the largest deviation $3.1 \sigma$ in the clean observable $\mathcal{R}_{K}$. In the following phenomenological analysis we are only considering the clean observables; namely, the ratios $\mathcal{R}_{K^{(*)}}$ and the branching fraction of the leptonic decays $\operatorname{Br}\left(B_{s} \rightarrow \ell^{+} \ell^{-}\right)$.

\section{A. Scalar leptoquark $\phi_{3}^{-2 / 3}$}

The scalar leptoquark $\phi_{3}^{-2 / 3}$ contributes to the following dimension six effective interactions:

$$
\mathcal{L}_{\text {eff }}^{\phi_{3}^{-2 / 3}} \supset \frac{4 G_{F}}{\sqrt{2}} V_{t b} V_{t s}^{*} \frac{\alpha}{4 \pi}\left[C_{9 \ell \ell}^{\prime}\left(\bar{s} \gamma_{\mu} P_{R} b\right)\left(\bar{\ell} \gamma^{\mu} \ell\right)+C_{10 \ell \ell}^{\prime}\left(\bar{s} \gamma_{\mu} P_{R} b\right)\left(\bar{\ell} \gamma^{\mu} \gamma^{5} \ell\right)\right]+\text { H.c., }
$$

whose Wilson coefficients are determined after integrating $\phi_{3}^{-2 / 3}$ out and are given by

$$
\begin{aligned}
C_{10 \ell \ell}^{\prime}=-C_{9 \ell \ell}^{\prime} & =\left(\frac{\sqrt{2} \pi}{G_{F} V_{t b} V_{t s}^{*} \alpha}\right) \frac{\left(K_{3}^{*} V_{\mathrm{PMNS}}^{*} V_{4}\right)^{\ell 3}\left(K_{3} V_{\mathrm{PMNS}} V_{4}^{*}\right)^{\ell 2}}{4 M_{\phi_{3}^{-2 / 3}}^{2}} \\
& \simeq(36 \mathrm{TeV})^{2} \frac{\left(K_{3}^{*} V_{\mathrm{PMNS}}^{*} V_{4}\right)^{\ell 3}\left(K_{3} V_{\mathrm{PMNS}} V_{4}^{*}\right)^{\ell 2}}{4 M_{\phi_{3}^{-2 / 3}}^{2}}
\end{aligned}
$$

The fact that the theory predicts $C_{10 \ell \ell}^{\prime}=-C_{9 \ell \ell}^{\prime}$ allows us to write the leptonic branching ratio $B_{s} \rightarrow \ell^{+} \ell^{-}$as a function of a single Wilson coefficient $C_{10 \ell \ell}^{\prime}$,

$$
\operatorname{Br}\left(B_{s} \rightarrow \ell^{+} \ell^{-}\right)=\operatorname{Br}\left(B_{s} \rightarrow \ell \ell\right)_{\mathrm{SM}} \times\left(1+0.4875 \operatorname{Re}\left[C_{10 \ell \ell}^{\prime}\right]+0.05940\left|C_{10 \ell \ell}^{\prime}\right|^{2}\right),
$$

where for the Standard Model prediction we take $\operatorname{Br}\left(B_{s} \rightarrow \mu^{+} \mu^{-}\right)_{\mathrm{SM}}=3.66 \times 10^{-9}$ [56] and $\operatorname{Br}\left(B_{s} \rightarrow e^{+} e^{-}\right)_{\mathrm{SM}}=$ $8.35 \times 10^{-14}[57,58]$. The same applies to the rest of the clean observables we are considering, ${ }^{3}$

$$
\begin{gathered}
\mathcal{R}_{K}=\mathcal{R}_{K}^{\mathrm{SM}} \frac{1-0.5040 \operatorname{Re}\left[C_{10 \mu \mu}^{\prime}\right]+0.06359\left|C_{10 \mu \mu}^{\prime}\right|^{2}}{1-0.5040 \operatorname{Re}\left[C_{10 e e}^{\prime}\right]+0.06359\left|C_{10 e e}^{\prime}\right|^{2}} \quad \text { for } q^{2} \subset[1.1,6] \mathrm{GeV}^{2}, \\
\mathcal{R}_{K^{*}}=\mathcal{R}_{K^{*}}^{\mathrm{SM}} \frac{1+0.4335 \operatorname{Re}\left[C_{10 \mu \mu}^{\prime}\right]+0.07473\left|C_{10 \mu \mu}^{\prime}\right|^{2}}{1+0.4325 \operatorname{Re}\left[C_{10 e e}^{\prime}\right]+0.07472\left|C_{10 e e}^{\prime}\right|^{2}} \quad \text { for } q^{2} \subset[1.1,6] \mathrm{GeV}^{2}, \\
\mathcal{R}_{K^{*}}=\mathcal{R}_{K^{*}}^{\mathrm{SM}} \frac{1+0.2363 \operatorname{Re}\left[C_{10 \mu \mu}^{\prime}\right]+0.03266\left|C_{10 \mu \mu}^{\prime}\right|^{2}}{1+0.2252 \operatorname{Re}\left[C_{10 e e}^{\prime}\right]+0.03127\left|C_{10 e e}^{\prime}\right|^{2}} \quad \text { for } q^{2} \subset[0.045,1.1] \mathrm{GeV}^{2} .
\end{gathered}
$$

For the contributions to the Wilson coefficients from the $\mathrm{SM}$ we take $C_{7}^{\mathrm{SM}}=-0.304, C_{9}^{\mathrm{SM}}=4.211$, and $C_{10}^{\mathrm{SM}}=$ -4.103 [61].

In Fig. 1 we show the parameter space in the $\operatorname{Br}\left(B_{s} \rightarrow\right.$ $\left.\mu^{+} \mu^{-}\right)$vs $\operatorname{Br}\left(B_{s} \rightarrow e^{+} e^{-}\right)$plane that satisfies the experimental value of $\mathcal{R}_{K}$ at the $1 \sigma$ level [10]; see Eq. (1). We note that, due to the quadratic dependence of the observables on $C_{10 \ell \ell}^{\prime}$, for a given $\operatorname{Br}\left(B_{s} \rightarrow \mu^{+} \mu^{-}\right)$and $\operatorname{Br}\left(B_{s} \rightarrow\right.$ $\left.e^{+} e^{-}\right)$there exist four possible values of $\mathcal{R}_{K}$ allowed. In this figure we present the solution that is also consistent with the measured window for $\mathcal{R}_{K}$. We also take into account the existing experimental bounds on the leptonic

\footnotetext{
${ }^{3}$ For the calculation of the ratios $\mathcal{R}_{K}$ and $\mathcal{R}_{K^{*}}$ we adopt the form factors from Ref. [59] and Ref. [60], respectively.
}

decay to muons [62] and to electrons [63], which are given by

$$
\begin{gathered}
\operatorname{Br}\left(B_{s} \rightarrow \mu^{+} \mu^{-}\right)^{\exp }=3.0 \pm 0.6_{-0.2}^{+0.3} \times 10^{-9} \\
\operatorname{Br}\left(B_{s} \rightarrow e^{+} e^{-}\right)^{\exp }<2.8 \times 10^{-7}
\end{gathered}
$$

The region shaded in gray in Fig. 1 shows explicitly the parameter space satisfying $\operatorname{Br}\left(B_{s} \rightarrow \mu^{+} \mu^{-}\right)^{\exp }$ [62] at the $1 \sigma$ level. As the figure shows, we find that there is a region of the parameter space that satisfies $\mathcal{R}_{K}^{\exp }$ and $\operatorname{Br}\left(B_{s} \rightarrow\right.$ $\left.\mu^{+} \mu^{-}\right)^{\exp }$ at $1 \sigma$ which corresponds to the overlapping region between the regions shaded in gray and in orange in the plot. 
In Fig. 2 we plot the correlation between the semileptonic ratios for the different $q^{2}$ ranges tested at experiment for the values of $\operatorname{Br}\left(B_{s} \rightarrow \mu^{+} \mu^{-}\right)$and $\operatorname{Br}\left(B_{s} \rightarrow e^{+} e^{-}\right)$consistent with the experimental values of $\mathcal{R}_{K}^{\exp }$ and $\operatorname{Br}\left(B_{s} \rightarrow \mu^{+} \mu^{-}\right)^{\exp }$ at the $1 \sigma$ level. We note that the theory predicts a window for $\mathcal{R}_{K^{*}}$ that is consistent with the experimental values of this observable [64],

$$
\mathcal{R}_{K^{*}}^{\exp }= \begin{cases}0.66_{-0.07}^{+0.11}(\text { stat }) \pm 0.03(\text { syst }) & \text { for } 0.045<q^{2}<1.1 \mathrm{GeV}^{2} \\ 0.69_{-0.07}^{+0.11}(\text { stat }) \pm 0.05(\text { syst }) & \text { for } 1.1<q^{2}<6.0 \mathrm{GeV}^{2}\end{cases}
$$

which deviate from the Standard Model prediction by $2.2 \sigma$ and $2.4 \sigma$, respectively. Since the main focus of our work is to explain $\mathcal{R}_{K}$ and $\operatorname{Br}\left(B_{s} \rightarrow \mu^{+} \mu^{-}\right)$, we aim to reproduce

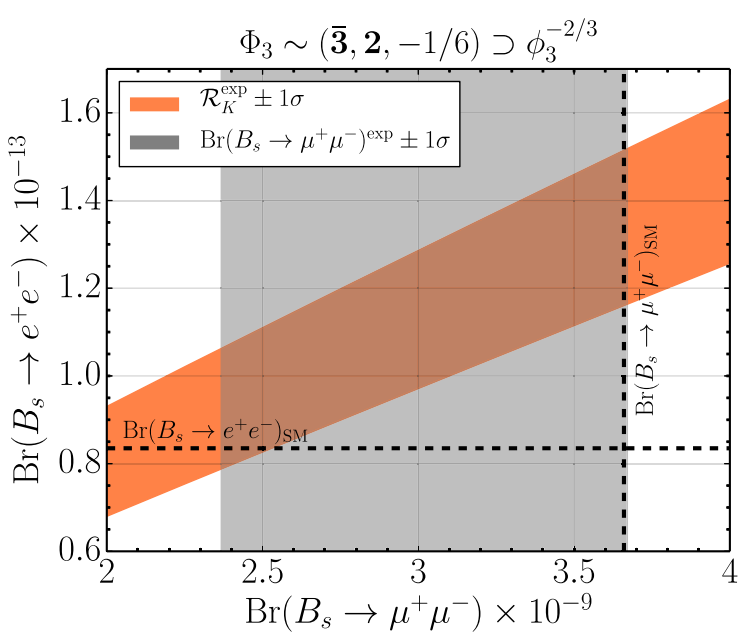

FIG. 1. The orange band gives the correlation between $\operatorname{Br}\left(B_{s} \rightarrow e^{+} e^{-}\right)$and $\operatorname{Br}\left(B_{s} \rightarrow \mu^{+} \mu^{-}\right)$that explains the $\mathcal{R}_{K}$ experimental measurement within $1 \sigma$. The gray band corresponds to the measurement of $\operatorname{Br}\left(B_{s} \rightarrow \mu^{+} \mu^{-}\right)$within $1 \sigma$. The black dashed lines correspond to the SM predictions for each channel. these observables within $1 \sigma$, while for $\mathcal{R}_{K^{*}}$ we consider the $2 \sigma$ ranges. As Fig. 2 shows, for $\mathcal{R}_{K^{*}}$ in the range $1.1<$ $q^{2}<6 \mathrm{GeV}^{2}$ the full predicted window is in agreement with the experimental measurement within $1 \sigma$, while for $0.045<q^{2}<1.1 \mathrm{GeV}^{2}$, the theory prefers higher values still within $2 \sigma$ of its experimental value.

Figure 3 shows the parameter space in the plane of the relevant Wilson coefficients $C_{10 \mu \mu}^{\prime}$ and $C_{10 e e}^{\prime}$ that satisfies the experimental value of the clean observables at the $1 \sigma$ level: $\operatorname{Br}\left(B_{s} \rightarrow \mu^{+} \mu^{-}\right)^{\text {exp }}$ in orange and $\mathcal{R}_{K}^{\exp }$ in blue. For $\mathcal{R}_{K^{*}}^{[0.045,1.1]}$ and $\mathcal{R}_{K^{*}}^{[1.1,6]}$ we consider the $2 \sigma$ range of the measurements which are shown in red and green, respectively; between brackets we specify the window of the integrated $q^{2}$. As can be seen from the lower left part of the plot, all observables can be explained by Wilson coefficients $C_{10 \mu \mu}^{\prime} \approx-8$ and $C_{10 e e}^{\prime} \approx-9$.

As we have shown in this subsection, the simplest theory where one can understand unification of matter at the $\mathrm{TeV}$ scale naturally accommodates the so-called flavor anomalies in $b \rightarrow s$ transitions observed at experiment. As one can read from the Wilson coefficients in Eq. (20), such transitions particularly imply the presence of four entries in the Yukawa matrix between the charged leptons and the down quarks, which in the physical basis reads as
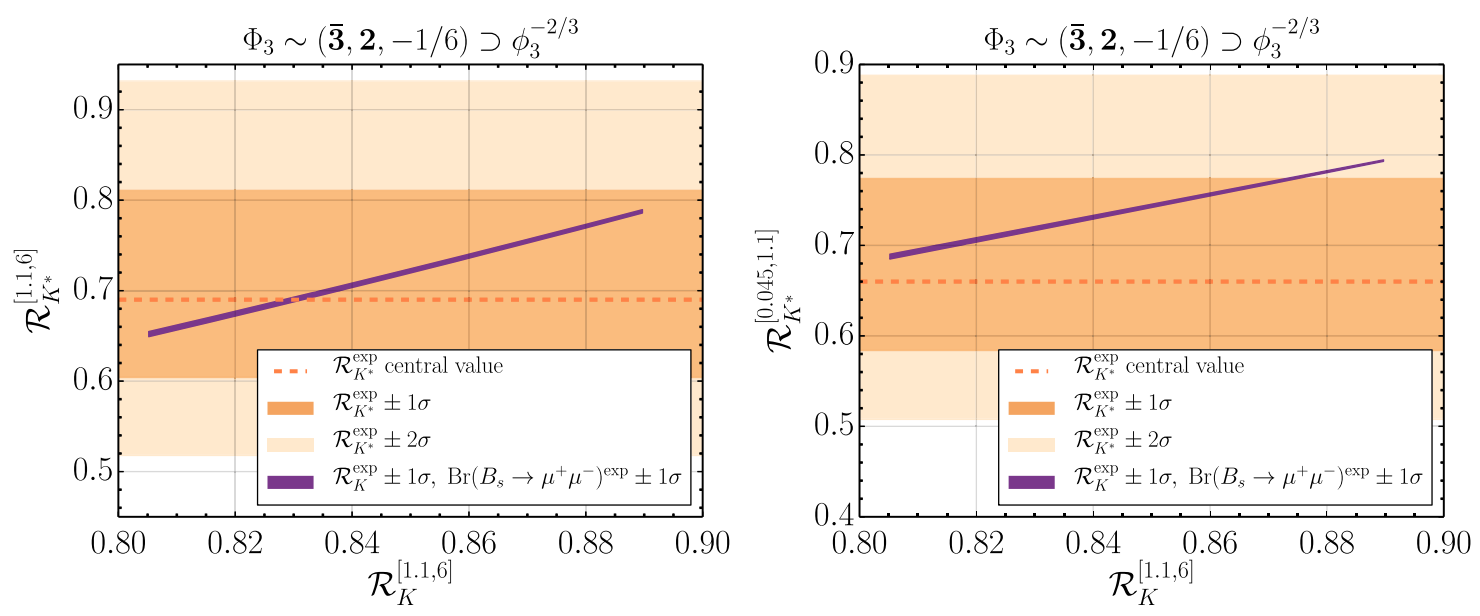

FIG. 2. The purple band gives the prediction for $\mathcal{R}_{K^{*}}$ in the window $0.045<q^{2}<1.1 \mathrm{GeV}^{2}$ (left panel) and $1.1<q^{2}<6 \mathrm{GeV}^{2}$ (right panel) for the points satisfying $\mathcal{R}_{K}^{\exp }$ and $\operatorname{Br}\left(B_{s} \rightarrow \mu^{+} \mu^{-}\right)$exp within $1 \sigma$. The region shaded in orange (light orange) corresponds to the measurement of $\mathcal{R}_{K^{*}}$ at $1 \sigma(2 \sigma)$. 


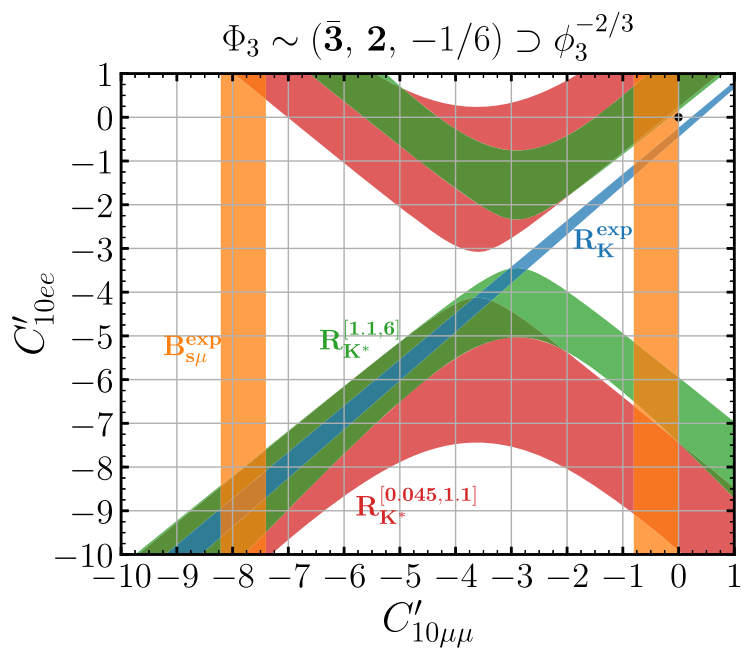

FIG. 3. Parameter space of the Wilson coefficients $C_{10 e e}^{\prime}$ and $C_{10 \mu \mu}^{\prime}$ required to explain the flavor anomalies. The orange band is in agreement with the measurement of $\operatorname{Br}\left(B_{s} \rightarrow \mu^{+} \mu^{-}\right)$and the blue band with $\mathcal{R}_{K}$ within $1 \sigma$. The red and green bands correspond to the measurements of $\mathcal{R}_{K^{*}}^{[0.045,1.1]}$ and $\mathcal{R}_{K^{*}}^{[1.1,6]}$ within $2 \sigma$, respectively.

$\tilde{V}_{4} \equiv K_{3}^{*} V_{\mathrm{PMNS}}^{*} V_{4}$, as can be read from Eq. (18). However, the rest of the couplings in this matrix may lead to other flavor transitions, such as $K_{L} \rightarrow \mu^{ \pm} e^{\mp}$ and $\tau$ decays to light mesons and a charged lepton, which suffer from strong experimental constraints. Requiring consistency with the experiment allows us to infer the texture of the Yukawa matrix $\tilde{V}_{4}$. By adopting the following hierarchy in their entries:

$$
\tilde{V}_{4}=\left(\begin{array}{ccc}
\cdot & \bigcirc & \bigcirc \\
\cdot & \bigcirc & \bigcirc \\
\cdot & \cdot & \cdot
\end{array}\right)
$$

where the squares denote large entries while the dots denote small entries, the theory can accommodate the experimental values of the clean observables involving $b \rightarrow s$ transitions while being consistent with all existing flavor constraints. Notice that lepton flavor violation processes such as the radiative decays $\mu \rightarrow e \gamma$ or the muon magnetic moment $(g-2)_{\mu}$ do not offer relevant constraints to the four matrix entries involved in $b \rightarrow s$ transitions since they suffer from the muon mass suppression and a near cancellation of the loop functions due to the leptoquark charge of $\left|Q_{\mathrm{LQ}}\right|=2 / 3$. We refer the reader to Appendix B for more details.

The leptoquark $\phi_{3}^{-2 / 3}$ can also induce the decays $B \rightarrow$ $K \mu^{ \pm} e^{\mp}$ and $B_{s} \rightarrow \mu^{ \pm} e^{\mp}$; however, these decays depend on a different combination of couplings than the ones that enter in $\mathcal{R}_{K^{(*)}}$, and hence, there exists enough freedom in the Wilson coefficients for this decay to satisfy the experimental constraint. Furthermore, $C_{10}^{\prime}$ will also modify the $B_{s}-\bar{B}_{s}$ mass difference at one-loop that leads to the constraint $\left|C_{10}^{\prime}\right| M_{\phi_{3}^{2 / 3}} \lesssim 100 \mathrm{TeV}$ [65]. Since we need $C_{10}^{\prime} \simeq-9$ to explain the flavor anomalies, the mass of the leptoquark must satisfy $M_{\phi_{3}^{2 / 3}} \lesssim 10 \mathrm{TeV}$.

On the other hand, we note that $\phi_{3}^{1 / 3}$, which also belongs to the $\mathrm{SU}(2)_{L}$ doublet in Eq. (18), shares the entries of $\tilde{V}_{4}$ up to the effect of the $V_{\text {PMNS }}$ and some complex diagonal phases. Knowing the texture of $\tilde{V}_{4}$ from the interactions involving $\phi_{3}^{-2 / 3}$, the theory predicts a modification of processes such as $B \rightarrow K^{(*)} \nu \bar{\nu}$ and $B_{s} \rightarrow \nu \bar{\nu}$. The current experimental bound on the former is given by $\operatorname{Br}\left(B^{+} \rightarrow\right.$ $\left.K^{+} \nu \bar{\nu}\right)<1.7 \times 10^{-5}[66]$, which is a factor of 3 larger than the prediction in the $\mathrm{SM} \mathrm{Br}\left(B^{+} \rightarrow K^{+} \nu \bar{\nu}\right)_{\mathrm{SM}}=5.6 \times 10^{-6}$ [67], and $\operatorname{Br}\left(B^{+} \rightarrow K^{*+} \nu \bar{\nu}\right)<4 \times 10^{-5}$ [68], still far from the SM prediction $\operatorname{Br}\left(B^{+} \rightarrow K^{*+} \nu \bar{\nu}\right)_{\mathrm{SM}}=9.6 \times 10^{-6}$ [67]. Because of this and the large uncertainties in the hadronic form factors, it is hard to obtain constraints from these observables.

\section{B. Scalar leptoquark $\phi_{4}^{2 / 3}$}

The scalar leptoquark $\phi_{4}^{2 / 3}$ contributes to the following dimension six effective interactions:

$$
\mathcal{L}_{\text {eff }}^{\phi_{4}^{2 / 3}}=\frac{4 G_{F}}{\sqrt{2}} V_{t b} V_{t s}^{*} \frac{\alpha}{4 \pi}\left[C_{9 \ell \ell}\left(\bar{s} \gamma_{\mu} P_{L} b\right)\left(\bar{\ell} \gamma^{\mu} \ell\right)+C_{10 \ell \ell}\left(\bar{s} \gamma_{\mu} P_{L} b\right)\left(\bar{\ell} \gamma^{\mu} \gamma_{5} \ell\right)\right]+\text { H.c. }
$$

where the Wilson coefficients are given by

$$
C_{10 \ell \ell}=C_{9 \ell \ell}=-\left(\frac{\pi \sqrt{2}}{G_{F} V_{t b} V_{t s}^{*} \alpha}\right) \frac{\left(K_{2} V_{\mathrm{CKM}}^{T} K_{1} V_{6}\right)^{3 \ell}\left(K_{2}^{*} V_{\mathrm{CKM}}^{\dagger} K_{1}^{*} V_{6}^{*}\right)^{2 \ell}}{4 M_{\phi_{4}^{2 / 3}}^{2}} .
$$

In this case the leptonic branching ratio is also given as a function of a single Wilson coefficient, $C_{10 \ell \ell}$,

$$
\operatorname{Br}\left(B_{s} \rightarrow \ell^{+} \ell^{-}\right)=\operatorname{Br}\left(B_{s} \rightarrow \ell^{+} \ell^{-}\right)_{\mathrm{SM}} \times\left(1-0.487448 \operatorname{Re}\left[C_{10 \ell \ell}\right]+0.0594014\left|C_{10 \ell \ell}\right|^{2}\right),
$$



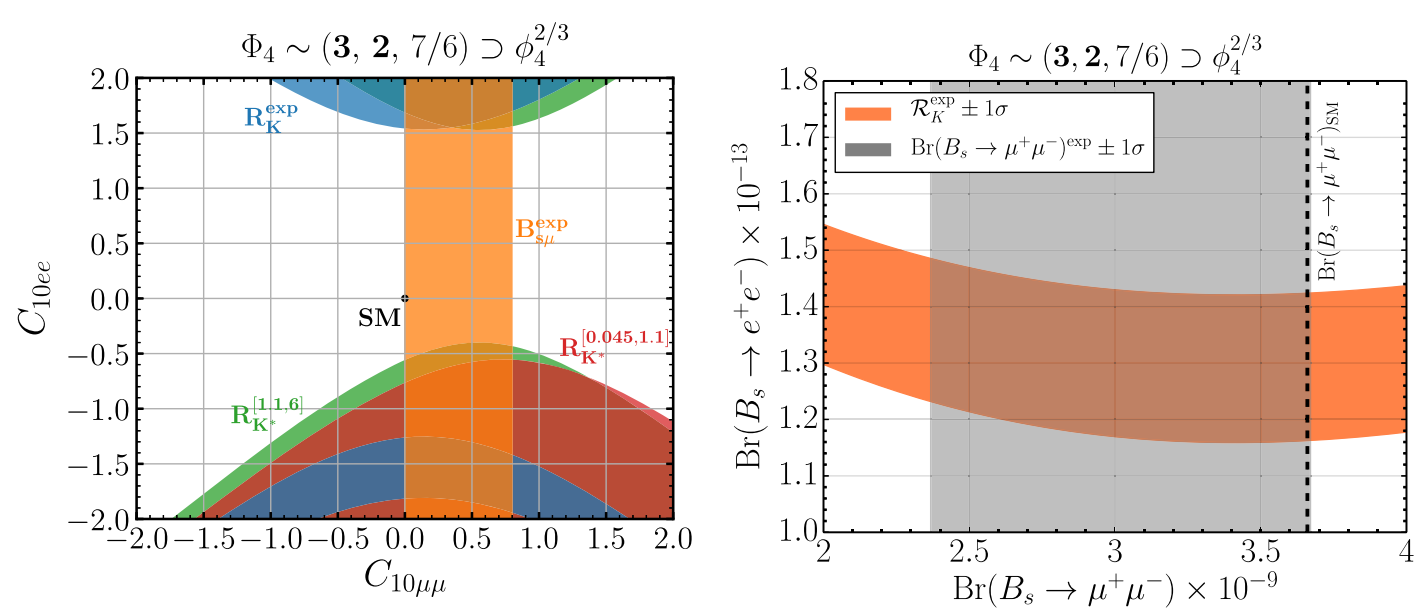

FIG. 4. Left panel: Parameter space of the Wilson coefficients $C_{10 e e}$ and $C_{10 \mu \mu}$ required to explain the flavor anomalies. The orange band is in agreement with the measurement of $\operatorname{Br}\left(B_{s} \rightarrow \mu^{+} \mu^{-}\right)$and the blue band with $\mathcal{R}_{K}$ within $1 \sigma$. The red and green bands correspond to the measurements of $\mathcal{R}_{K^{*}}^{[0.045,1.1]}$ and $\mathcal{R}_{K^{*}}^{[1.1,6]}$ within $2 \sigma$, respectively. Right panel: The orange band gives the correlation between $\operatorname{Br}\left(B_{s} \rightarrow e^{+} e^{-}\right)$and $\operatorname{Br}\left(B_{s} \rightarrow \mu^{+} \mu^{-}\right)$that explains the $\mathcal{R}_{K}$ experimental measurement within $1 \sigma$. The gray band corresponds to the measurement of $\operatorname{Br}\left(B_{s} \rightarrow \mu^{+} \mu^{-}\right)$within $1 \sigma$. In both panels, only the region where $C_{10 \mu \mu}$ is very close to zero is allowed by the $\mu \rightarrow e \gamma$ constraint. Therefore, the prediction for $\operatorname{Br}\left(B_{s} \rightarrow \mu^{+} \mu^{-}\right)$is very close to the SM prediction; the latter is shown by a black dashed line.

as well as the other clean observables we consider,

$$
\begin{gathered}
\mathcal{R}_{K}=\mathcal{R}_{K}^{\mathrm{SM}} \frac{1-0.01812 \operatorname{Re}\left[C_{10 \mu \mu}\right]+0.06359\left|C_{10 \mu \mu}\right|^{2}}{1-0.01781 \operatorname{Re}\left[C_{10 e e}\right]+0.06359\left|C_{10 e e}\right|^{2}} \quad \text { for } q^{2} \subset[1.1,6] \mathrm{GeV}^{2}, \\
\mathcal{R}_{K^{*}}=\mathcal{R}_{K^{*}}^{\mathrm{SM}} \frac{1-0.08301 \operatorname{Re}\left[C_{10 \mu \mu}\right]+0.07473\left|C_{10 \mu \mu}\right|^{2}}{1-0.08428 \operatorname{Re}\left[C_{10 e e}\right]+0.07472\left|C_{10 e e}\right|^{2}} \quad \text { for } q^{2} \subset[1.1,6] \mathrm{GeV}^{2}, \\
\mathcal{R}_{K^{*}}=\mathcal{R}_{K^{*}}^{\mathrm{SM}} \frac{1-0.04783 \operatorname{Re}\left[C_{10 \mu \mu}\right]+0.03266\left|C_{10 \mu \mu}\right|^{2}}{1-0.04600 \operatorname{Re}\left[C_{10 e e}\right]+0.03127\left|C_{10 e e}\right|^{2}} \quad \text { for } q^{2} \subset[0.045,1.1] \mathrm{GeV}^{2} .
\end{gathered}
$$
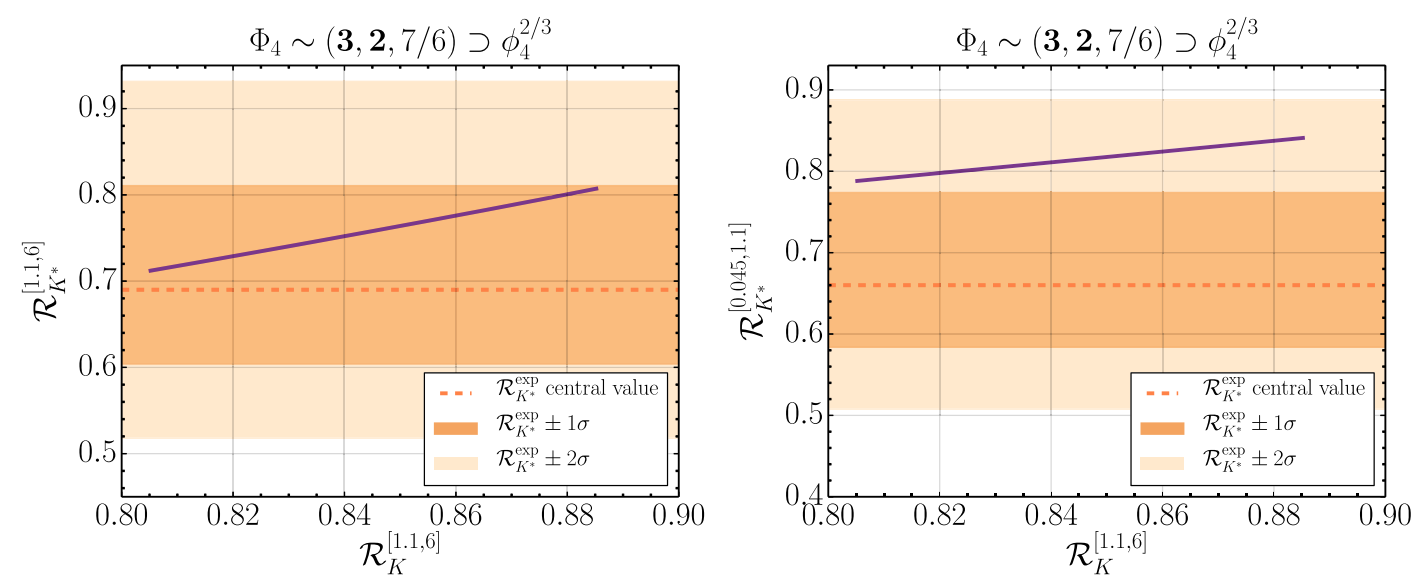

FIG. 5. The purple band gives the prediction for $\mathcal{R}_{K^{*}}$ in the window $0.045<q^{2}<1.1 \mathrm{GeV}^{2}$ (left panel) and $1.1<q^{2}<6 \mathrm{GeV}^{2}$ (right panel) for the points satisfying $\mathcal{R}_{K}^{\exp }$ within $1 \sigma$. The region shaded in orange (light orange) corresponds to the measurement of $\mathcal{R}_{K^{*}}$ at $1 \sigma(2 \sigma)$. We show the solution with small Wilson coefficients. 
In the left panel in Fig. 4 we show our results in the plane of the Wilson coefficients $C_{10 e e}$ vs $C_{10 \mu \mu}$. The orange band reproduces the measured value for $\operatorname{Br}\left(B_{s} \rightarrow \mu^{+} \mu^{-}\right)$while the blue band reproduces $\mathcal{R}_{K}$ within $1 \sigma$. The red and green bands reproduce the measurements of $\mathcal{R}_{K^{*}}^{[0.045,1.1]}$ and $\mathcal{R}_{K^{*}}^{[1.1,6]}$ within $2 \sigma$, respectively. In contrast to the $\Phi_{3}$ leptoquark, $\Phi_{4}$ can reproduce the observables with small Wilson coefficients and coupling mainly to electrons; namely, $C_{10 \mu \mu} \approx 0$ and $C_{10 e e} \approx-1.3$. Therefore they will have a smaller impact on each separate channel. Note that the component $\phi_{4}^{5 / 3}$ can give large contributions to $\mu \rightarrow e \gamma$ since the near cancellation on the loop functions that takes place for $\phi_{4}^{2 / 3}$ does not take place for $\phi_{4}^{5 / 3}$; see Appendix B for more details. Therefore, in order to be consistent with the lepton flavor violation constraints, the following texture in the Yukawa matrix $\tilde{V}_{6}=K_{2} V_{\mathrm{CKM}}^{T} K_{1} V_{6}$ must be adopted,

$$
\tilde{V}_{6}=\left(\begin{array}{lll}
\dot{0} & \cdot & \cdot \\
\bigcirc & \cdot & \cdot
\end{array}\right),
$$

where the leptoquark $\Phi_{4}$ couples mostly to electrons.

In Fig. 5 we show the correlation predicted for the ratios $\mathcal{R}_{K^{*}}$ and $\mathcal{R}_{K}$ adopting the texture in Eq. (35). In the left panel we give the predictions ${ }^{4}$ for $\mathcal{R}_{K^{*}}$ in the window $1.1<q^{2}<6 \mathrm{GeV}^{2}$, where the purple band is in agreement with $\mathcal{R}_{K}$ within $1 \sigma$. In the right panel we show the predictions for $\mathcal{R}_{K^{*}}$ in the window $0.045<q^{2}<1.1 \mathrm{GeV}^{2}$.

\section{THE $g-2$ OF THE MUON}

The Fermilab $g-2$ experiment has recently reported results on the anomalous magnetic moment of the muon $a_{\mu} \equiv(g-2)_{\mu} / 2$ from their Run 1 [36]. The combined result with the one from the E821 experiment at BNL [37] deviates from the SM prediction by $4.2 \sigma$, as Eq. (2) manifests. In this section we show that in the most general case, the theory gives a prediction for $(g-2)_{\mu}$ that can explain the reported deviation, involving the same leptoquarks in the theory that have been discussed so far.

In this theory, the main contribution to the muon $g-2$ is generated by the scalar leptoquark $\phi_{4}^{5 / 3}$ with the top quark running in the loop. In Fig. 6 we show the Feynman graphs for the two different topologies. The relevant Yukawa interactions for the $\phi_{4}^{5 / 3}$ field are given by

$$
-\mathcal{L} \supset \bar{e}^{i}\left(\lambda_{R}^{i j} P_{L}+\lambda_{L}^{i j} P_{R}\right) u^{j}\left(\phi_{4}^{5 / 3}\right)^{*}+\text { H.c. },
$$

\footnotetext{
${ }^{4}$ We note that the experimental value $\operatorname{Br}\left(B_{s} \rightarrow \mu^{+} \mu^{-}\right)^{\exp }$ is already consistent with the SM prediction at $1 \sigma$.
}
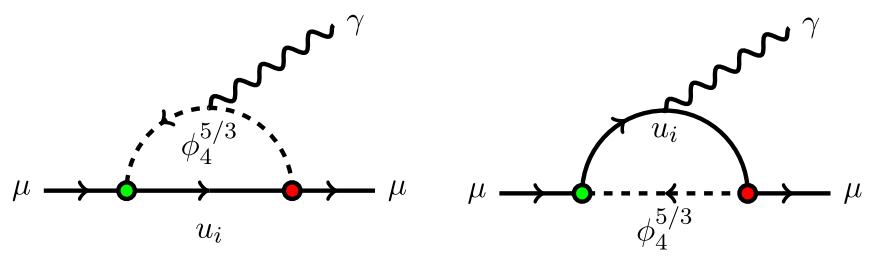

FIG. 6. Feynman diagrams for the topologies of the main contributions from the scalar leptoquark $\phi_{4}^{5 / 3}$ to $(g-2)_{\mu}$. The different colors in the vertices indicate opposite chiralities in the leptoquark-muon coupling.

where the matrices $\lambda_{L, R}$ correspond to

$$
\lambda_{R}=V_{6}^{T} \quad \text { and } \quad \lambda_{L}=-K_{3} V_{\mathrm{PMNS}} V_{5}^{*},
$$

and these matrices can be written in terms of the Yukawa matrices in the Lagrangian as $V_{5}=N^{T} Y_{2} U_{C}$ and $V_{6}=$ $U^{T} Y_{4} E_{C}$ as discussed in Appendix A. The new contribution to the muon $g-2$ can be written as

$$
\begin{aligned}
\Delta a_{\mu}= & \frac{-3}{16 \pi^{2}} \frac{m_{\mu}^{2}}{M_{\phi_{4}^{5 / 3}}^{2}} \sum_{j}\left[\left(\left|\lambda_{L}^{2 j}\right|^{2}+\left|\lambda_{R}^{2 j}\right|^{2}\right)\right. \\
& \times\left(\frac{2}{3} F_{1}\left(x_{j}\right)+\frac{5}{3} F_{2}\left(x_{j}\right)\right) \\
& \left.+\frac{m_{u_{j}}}{m_{\mu}} \operatorname{Re}\left[\lambda_{L}^{2 j}\left(\lambda_{R}^{2 j}\right)^{*}\right]\left(\frac{2}{3} F_{3}\left(x_{j}\right)+\frac{5}{3} F_{4}\left(x_{j}\right)\right)\right],
\end{aligned}
$$

where the loop functions are given by

$$
\begin{gathered}
F_{1}(x)=\frac{1}{6(1-x)^{4}}\left(2+3 x-6 x^{2}+x^{3}+6 x \ln x\right), \\
F_{2}(x)=\frac{1}{6(1-x)^{4}}\left(1-6 x+3 x^{2}+2 x^{3}-6 x^{2} \ln x\right), \\
F_{3}(x)=\frac{1}{(1-x)^{3}}\left(-3+4 x-x^{2}-2 \ln x\right), \\
F_{4}(x)=\frac{1}{(1-x)^{3}}\left(1-x^{2}+2 x \ln x\right),
\end{gathered}
$$

with $x_{j}=\left(m_{u_{j}} / M_{\phi_{4}^{5 / 3}}\right)^{2}$. Therefore, the dominant contribution will come from the top quark in the loop.

We present our results in Fig. 7 in the $\lambda_{L}^{23}$ vs $M_{\phi_{4}^{5 / 3}}$ plane.

The shaded bands are in agreement with the combined result from the Muon $g-2$ experiment at Fermilab and E821 at BNL within $1 \sigma$ given in Eq. (2). The orange band corresponds to fixing $\lambda_{R}^{23}=-\lambda_{L}^{23} / 5$, the blue band is for $\lambda_{R}^{23}=-\lambda_{L}^{23}$, while the green band is for $\lambda_{R}^{23}=-5 \lambda_{L}^{23}$.

The contributions from $\phi_{3}^{-2 / 3}$ and $\phi_{4}^{2 / 3}$ to the muon $g-2$ have chiral suppression and although this can be lifted through mixing, the latter is determined by the electroweak 


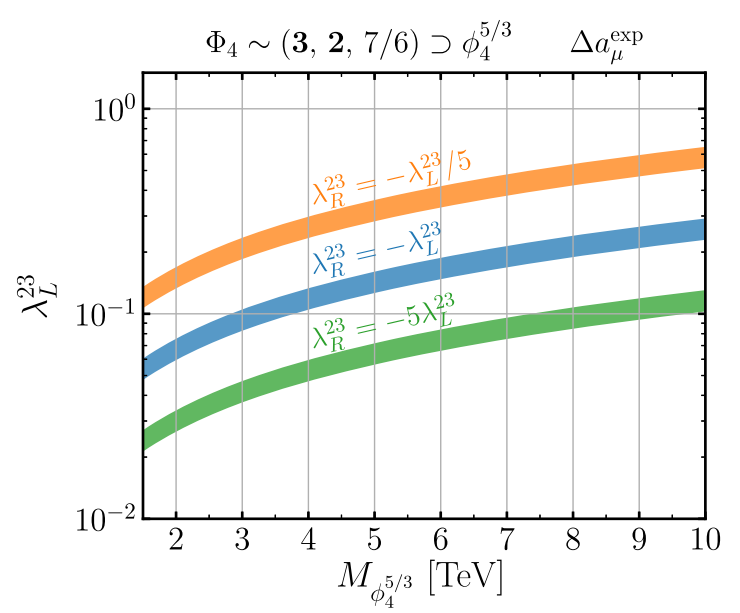

FIG. 7. Parameter space in the $\lambda_{L}^{23}$ vs $M_{\phi^{5 / 3}}$ plane, the shaded regions reproduce the combined result from the Muon $g-2$ experiment at Fermilab and E821 at BNL within $1 \sigma$. Different colors correspond to different relations between the couplings $\lambda_{L}^{23}$ and $\lambda_{R}^{23}$.

scale and generically it is very small; furthermore, the constraints from the LHC rule out such a scenario as an explanation for the $(g-2)_{\mu}$ anomaly [44].

\section{A. Connection between the flavor and muon $g-2$ anomalies}

In this section we study the possibility to explain the $\mathcal{R}_{K}$ and the $(g-2)_{\mu}$ anomalies simultaneously. For alternative solutions to both of these anomalies see e.g., Refs. [69-74]. In the theory discussed in this work, the flavor and muon $g-2$ anomalies can be simultaneously explained when we consider the contributions from both $\Phi_{3}$ and $\Phi_{4}$. As we have discussed, $\Phi_{4}$ can explain the $g-2$ of the muon, and in order to avoid the strong experimental constraint on

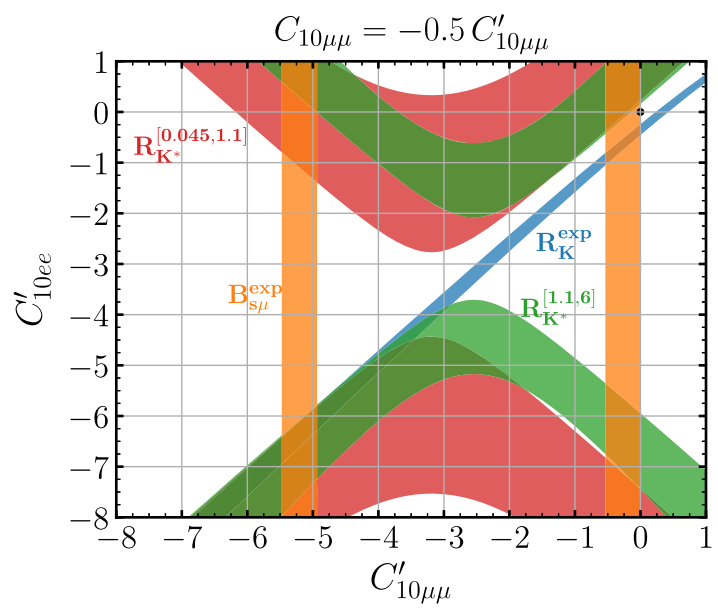

$\mu \rightarrow e \gamma$ we assume that $\Phi_{4}$ couples mainly to muons by adopting the following texture:

$$
\tilde{V}_{6}=K_{2} V_{\mathrm{CKM}}^{T} K_{1} V_{6}=\left(\begin{array}{lll}
\cdot & \dot{1} & \cdot \\
\cdot & \bigcirc & \cdot \\
\cdot & \bigcirc & \cdot
\end{array}\right) .
$$

As we studied in Sec. III A, $\Phi_{3}$ can be used to explain the flavor anomalies by being coupled to both electrons and muons, and hence, we assume the following texture:

$$
\tilde{V}_{4}=K_{3}^{*} V_{\mathrm{PMNS}}^{*} V_{4}=\left(\begin{array}{ccc}
\cdot & \bigcirc & \bigcirc \\
\cdot & \bigcirc & \bigcirc \\
\cdot & \cdot & \cdot
\end{array}\right)
$$

As given in Eq. (37) the coupling $\lambda_{L}^{23}$ is determined by the matrix $V_{5}$. Therefore, for the matrix $V_{5}$ we only require the entry $V_{5}^{23}$ to be nonzero.

In the left panel in Fig. 8 we show the parameter space of the Wilson coefficients $C_{10 e e}^{\prime}, C_{10 \mu \mu}^{\prime}$, and $C_{10 \mu \mu}$, assuming that $\Phi_{4}$ couples mainly to muons [i.e., adopting the texture in Eq. (43)]. As can be seen in this plot, the flavor observables can be reproduced with $C_{10 e e}^{\prime} \approx-6$, $C_{10 \mu \mu}^{\prime} \approx-5$, and for the contribution from $\Phi_{4}$ we take $C_{10 \mu \mu}=-0.5 C_{10 \mu \mu}^{\prime}$.

The entries in the $V_{6}$ matrix can be written in terms of the coefficient $C_{10 \mu \mu}$ as follows:

$$
V_{6}^{32}\left(V_{6}^{22}\right)^{*}=-4 M_{\phi_{4}^{2 / 3}}^{2} C_{10 \mu \mu}\left(\frac{G_{F} V_{t b} V_{t s}^{*} \alpha}{\pi \sqrt{2}}\right)
$$

where we have assumed $K_{2} V_{\mathrm{CKM}} K_{1} \sim \mathbf{1}$, for simplicity. Notice that one of the couplings, $\lambda_{R}^{23}$, needed to predict $(g-2)_{\mu}$ is $\lambda_{R}^{23}=-V_{6}^{32}$. Then, assuming $V_{6}^{32}=-V_{6}^{22}$, the

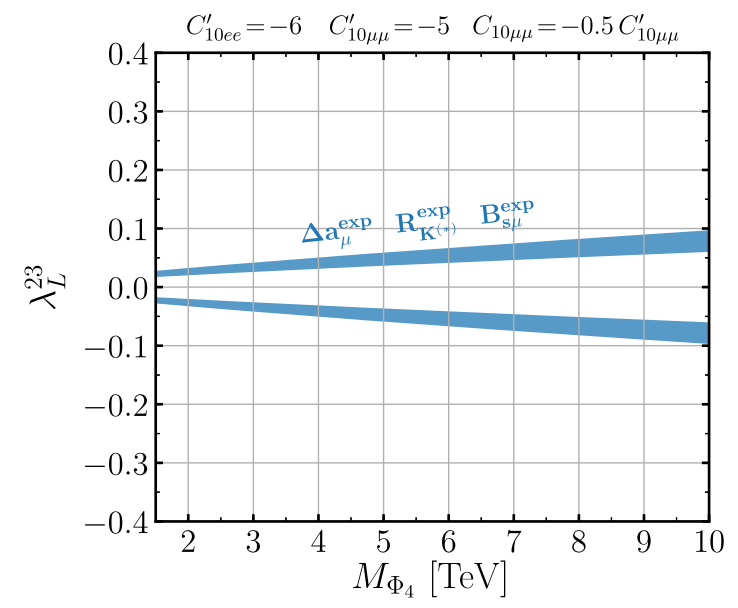

FIG. 8. Left panel: Same as Fig. 3; we also include a contribution from $C_{10 \mu \mu}=-0.5 C_{10 \mu \mu}^{\prime}$. Right panel: The region shaded in blue is in agreement with the combined result from the Muon $g-2$ experiment at Fermilab and E821 at BNL within $1 \sigma$. We have also fixed the Wilson coefficients that reproduce the experimental measurements of $\operatorname{Br}\left(B_{s} \rightarrow \mu^{+} \mu^{-}\right)$and $\mathcal{R}_{K}$ within $1 \sigma$ and $\mathcal{R}_{K^{*}}$ within $2 \sigma$. 

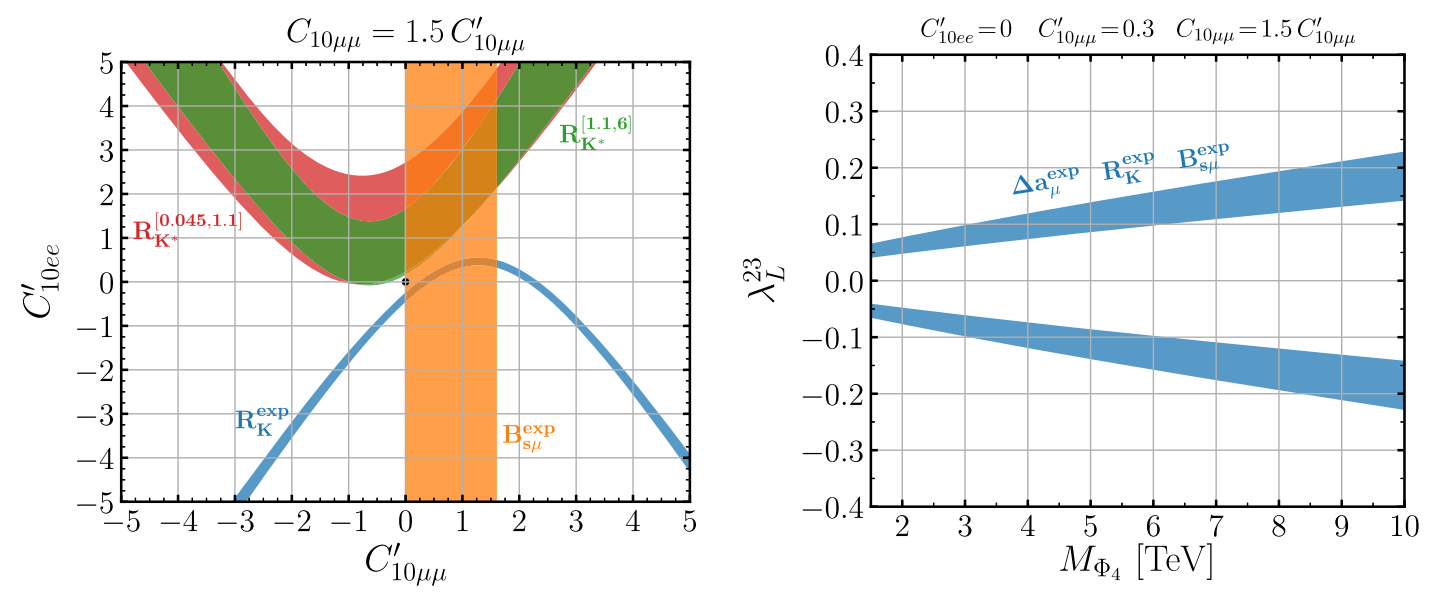

FIG. 9. Left panel: Same as Fig. 3; we also include a contribution from $C_{10 \mu \mu}=1.5 C_{10 \mu \mu}^{\prime}$. Right panel: The region shaded in blue is in agreement with the combined result from the Muon $g-2$ experiment at Fermilab and E821 at BNL within $1 \sigma$. We have also fixed the Wilson coefficients that reproduce the experimental measurements of $\operatorname{Br}\left(B_{s} \rightarrow \mu^{+} \mu^{-}\right)$and $\mathcal{R}_{K}$ within $1 \sigma$. The predicted values for $\mathcal{R}_{K^{*}}$ are higher than the current central values as discussed in the text.

coupling $\lambda_{R}^{23}$ can be written as a function of $M_{\phi_{4}^{2 / 3}}$ using the above equation. Neglecting the mass splitting between the fields in $\Phi_{4}$, we have $M_{\phi_{4}^{2 / 3}}=M_{\phi_{4}^{5 / 3}}=M_{\Phi_{4}}$. Therefore, the predictions for $g-2$ will depend only on two parameters, $\lambda_{L}^{23}$ and $M_{\Phi_{4}}$. In the right panel in Fig. 8 we show the predictions for the Muon $g-2$ after fixing the Wilson coefficients to the values that reproduce the flavor anomalies. The blue band is in agreement with the measured value of $(g-2)_{\mu}$ within $1 \sigma$. Consequently, there are consistent scenarios that provide a simultaneous explanation of the flavor and muon $g-2$ anomalies.

There also exists a solution with smaller values for the Wilson coefficients even though the measured values for $\mathcal{R}_{K^{*}}$ cannot be reproduced. First of all, for $\phi_{4}^{5 / 3}$ to be able to explain $(g-2)_{\mu}$ at the multi-TeV scale we need the coefficient $C_{10 \mu \mu}$ to be order one, so we set $C_{10 \mu \mu}=1.5 C_{10 \mu \mu}^{\prime}$. From the left panel in Fig. 9 we see that for $C_{10 \mu \mu}^{\prime}=0.3$ and $C_{10 e e}^{\prime}=0$ we can reproduce the measured values for $\mathcal{R}_{K}$ and $\operatorname{Br}\left(B_{s} \rightarrow \mu^{+} \mu^{-}\right)$; moreover, in this scenario the new physics is only coupled to muons. However, the predictions for $\mathcal{R}_{K^{*}}$ turn out to be larger than the current observed value. In the right panel of Fig. 9 we assume that $V_{6}^{32}=-V_{6}^{22}$ and show the region in the $\lambda_{L}$ vs $M_{\Phi_{4}}$ plane that explains the $g-2$ of the muon within $1 \sigma$.

Focusing on the region that reproduces $\operatorname{Br}\left(B_{s} \rightarrow \mu^{+} \mu^{-}\right)$ and $\mathcal{R}_{K}$ within $1 \sigma$, in the left panel of Fig. 9, we obtain the following predictions for $\mathcal{R}_{K^{*}}: \mathcal{R}_{K^{*}}^{[0.045,1.1]}=[0.98-1.34]$, $\mathcal{R}_{K^{*}}^{[1.1,6]}=[1.08-1.85]$, both are much higher than the current observed values given in Eq. (27). The values of the Wilson coefficients on the right panel in Fig. 9 correspond to the lower end in these ranges.

\section{SUMMARY}

We have discussed the simplest quark-lepton unification theory that can be realized at the $\mathrm{TeV}$ scale [6] and can be seen as a low energy limit of the Pati-Salam theory. This theory is based on the $\mathrm{SU}(4)_{C} \otimes \mathrm{SU}(2)_{L} \otimes \mathrm{U}(1)_{R}$ gauge group and, in order to have a consistent theory for fermion masses at the low scale, neutrino masses are generated through the inverse seesaw mechanism. This theory predicts the existence of a vector leptoquark, $X_{\mu} \sim(\mathbf{3}, \mathbf{1}, 2 / 3)_{\mathrm{SM}}$, and two scalar leptoquarks, $\Phi_{3} \sim$ $(\overline{\mathbf{3}}, \mathbf{2},-1 / 6)_{\mathrm{SM}}$ and $\Phi_{4} \sim(\mathbf{3}, \mathbf{2}, 7 / 6)_{\mathrm{SM}}$, that can provide a relevant contribution to meson decays.

We have studied the possibility to explain the experimental values for the clean observables involving $b \rightarrow s$ transitions, i.e., $\mathcal{R}_{K}, \mathcal{R}_{K^{*}}$ and $\operatorname{Br}\left(B_{s} \rightarrow \mu^{+} \mu^{-}\right)$, in two main scenarios. In the first scenario the scalar leptoquark $\Phi_{3}$ gives the main contributions to explain the measured values of the relevant meson decays through couplings to both electrons and muons. In the second scenario the scalar leptoquark $\Phi_{4}$ plays the main role to explain the values for the neutral flavor anomalies; in this scenario the new physics is coupled mostly to electrons as it is required by the experimental bound from $\mu \rightarrow e \gamma$. Furthermore, we showed that $\Phi_{4}$ can be used to explain the $g-2$ of the muon while being consistent with other experimental bounds.

We found scenarios where we can address simultaneously the flavor and the $(g-2)_{\mu}$ anomalies, in which both leptoquarks $\Phi_{3}$ and $\Phi_{4}$ play a role. In these scenarios, the recent experimental results for $\mathcal{R}_{K}$ and $\mathcal{R}_{K^{*}}$ are explained by contributions from $\Phi_{3}$ and $\Phi_{4}$, with Wilson coefficients of the same order as in the SM, while the measured value of $(g-2)_{\mu}$ can be addressed by coupling $\Phi_{4}$ mostly to muons, 
so that the aforementioned anomalies can all be explained in consistency with constraints from lepton flavor violation.

We hope that, in the near future, more experimental data and an improvement of the theoretical predictions will determine whether these anomalies represent final evidence for new physics, and whether the minimal theory for quarklepton unification can be behind them by contrasting alternative predictions with experimental results.

\section{ACKNOWLEDGMENTS}

We thank the referee for important feedback on an earlier version of this work. The work of P.F. P. has been supported by the U.S. Department of Energy, Office of Science, Office of High Energy Physics, under Award No. DE-SC0020443. The work of C. M. is supported by the U.S. Department of Energy, Office of Science, Office of
High Energy Physics, under Award No. DE-SC0011632 and by the Walter Burke Institute for Theoretical Physics.

\section{APPENDIX A: LEPTOQUARK INTERACTIONS}

In our convention the mass matrices are diagonalized as

$$
\begin{aligned}
& U^{T} M_{U} U_{C}=M_{U}^{\mathrm{diag}}, \\
& D^{T} M_{D} D_{C}=M_{D}^{\mathrm{diag}}, \\
& E^{T} M_{E} E_{C}=M_{E}^{\mathrm{diag}} .
\end{aligned}
$$

The following matrices enter in the leptoquark interactions below:

$$
\begin{aligned}
V_{1} & =N_{C}^{\dagger} U_{C}, \quad V_{2}=E_{C}^{\dagger} D_{C}, \quad V_{3}=U^{T} Y_{2} N_{C}, \quad V_{4}=N^{T} Y_{4} D_{C}, \quad V_{5}=N^{T} Y_{2} U_{C}, \quad \text { and } \quad V_{6}=U^{T} Y_{4} E_{C} ; \\
V_{\mathrm{DE}} & =D^{\dagger} E, \quad U^{\dagger} D=K_{1} V_{\mathrm{CKM}} K_{2}, \quad \text { and } \quad E^{\dagger} N=K_{3} V_{\mathrm{PMNS}} .
\end{aligned}
$$

$K_{1}$ and $K_{3}$ are diagonal matrices containing three phases, while $K_{2}$ has two phases.

(i) Vector leptoquark $X_{\mu} \sim(\mathbf{3}, \mathbf{1}, 2 / 3)_{\mathrm{SM}}$ :

$$
\begin{aligned}
& \frac{g_{4}}{\sqrt{2}} \bar{d}_{L} V_{\mathrm{DE}} \gamma^{\mu} e_{L} X_{\mu} \\
& \frac{g_{4}}{\sqrt{2}} \bar{u}_{L}\left(K_{1} V_{\mathrm{CKM}} K_{2} V_{\mathrm{DE}} K_{3} V_{\mathrm{PMNS}}\right) \gamma^{\mu} \nu_{L} X_{\mu}, \\
& \frac{g_{4}}{\sqrt{2}} \overline{\left(\nu^{c}\right)_{L}} V_{1} \gamma^{\mu}\left(u^{c}\right)_{L} X_{\mu}, \\
& \frac{g_{4}}{\sqrt{2}}{\overline{\left(e^{c}\right)_{L}}}_{L} V_{2} \gamma^{\mu}\left(d^{c}\right)_{L} X_{\mu} \text {. }
\end{aligned}
$$

(ii) Scalar leptoquark $\Phi_{3} \sim(\overline{\mathbf{3}}, \mathbf{2},-1 / 6)_{\mathrm{SM}}$ :

$$
\begin{gathered}
u_{L}^{T} C V_{3}\left(\nu^{c}\right)_{L} \phi_{3}^{-2 / 3}, \\
-d_{L}^{T} C K_{2} V_{\mathrm{CKM}}^{T} K_{1} V_{3}\left(\nu^{c}\right)_{L} \phi_{3}^{1 / 3}, \\
\nu_{L}^{T} C V_{4}\left(d^{c}\right)_{L}\left(\phi_{3}^{1 / 3}\right)^{*}, \\
e_{L}^{T} C K_{3}^{*} V_{\mathrm{PMNS}}^{*} V_{4}\left(d^{c}\right)_{L}\left(\phi_{3}^{-2 / 3}\right)^{*} .
\end{gathered}
$$

(iii) Scalar leptoquark $\Phi_{4} \sim(\mathbf{3}, \mathbf{2}, 7 / 6)_{\mathrm{SM}}$ :

$$
\begin{gathered}
\nu_{L}^{T} C V_{5}\left(u^{c}\right)_{L} \phi_{4}^{2 / 3}, \\
-e_{L}^{T} C K_{3}^{*} V_{\mathrm{PMNS}}^{*} V_{5}\left(u^{c}\right)_{L} \phi_{4}^{5 / 3},
\end{gathered}
$$

$$
\begin{gathered}
u_{L}^{T} C V_{6}\left(e^{c}\right)_{L}\left(\phi_{4}^{5 / 3}\right)^{*}, \\
d_{L}^{T} C K_{2} V_{\mathrm{CKM}}^{T} K_{1} V_{6}\left(e^{c}\right)_{L}\left(\phi_{4}^{2 / 3}\right)^{*} .
\end{gathered}
$$

Notice that when $Y_{2} \rightarrow 0$ the matrices $V_{3} \rightarrow 0$ and $V_{5} \rightarrow 0$.

\section{APPENDIX B: BOUNDS FROM $\mu \rightarrow e \gamma$}

In this Appendix we discuss the implications from the experimental bounds on $\mu \rightarrow e \gamma$ on the three scenarios studied in this work:

(i) $\boldsymbol{\Phi}_{3}$ scenario: In this case only the field $\phi_{3}^{-2 / 3}$ contributes to $\mu \rightarrow e \gamma$ but its contribution is chiral suppressed. Furthermore, the contribution from $\phi_{3}^{-2 / 3}$ has a near cancellation in the loop functions due to the electric charge of $-2 / 3$; this is because in the limit $x \rightarrow 0$ the loop functions approach $F_{1}(x) \rightarrow 1 / 3$ and $F_{2}(x) \rightarrow 1 / 6$, and hence, the combination $Q_{d} F_{1}(x)-Q_{\mathrm{LQ}} F_{2}(x) \approx \mathcal{O}(x)$ (i.e., it almost vanishes).

(ii) $\boldsymbol{\Phi}_{4}$ scenario: In this case two fields, $\phi_{4}^{2 / 3}$ and $\phi_{4}^{5 / 3}$, contribute to $\mu \rightarrow e \gamma$. The contribution from $\phi_{4}^{2 / 3}$ is suppressed as in the case of $\phi_{3}^{-2 / 3}$ discussed above. However, the component $\phi_{4}^{5 / 3}$ can still give a contribution to this observable larger than the current experimental bound. The decay width is given by $[75,76]$ 


$$
\begin{aligned}
\Gamma(\mu \rightarrow e \gamma) \simeq & \frac{\alpha}{4} \frac{m_{\mu}^{5}}{M_{\phi_{4}^{5 / 3}}^{4}} \sum_{j} \mid \frac{3}{32 \pi^{2}} \lambda_{R}^{2 j} \lambda_{R}^{1 j *} \\
& \times\left.\left[Q_{q} F_{1}\left(x_{j}\right)+Q_{\mathrm{LQ}} F_{2}\left(x_{j}\right)\right]\right|^{2},
\end{aligned}
$$

with $x_{j}=\left(m_{u_{j}} / M_{\phi_{4}^{5 / 3}}\right)^{2}, \lambda_{R}=V_{6}^{T}$, and assuming $V_{\text {CKM }} \sim 1$ we have that $\tilde{V}_{6} \simeq V_{6}$. In Fig. 10 we show the parameter space in the $\lambda_{R}$ vs $M_{\phi_{4}^{5 / 3}}$ plane. The region shaded in red is excluded by the experimental bound $\operatorname{Br}(\mu \rightarrow e \gamma) \leq 4.2 \times 10^{-13}$ [77]. This is the motivation behind the texture chosen in Eq. (35) with couplings mostly to electrons. For the plot we have chosen the benchmark values of $\lambda_{R}=\lambda_{R}^{23}=-\lambda_{R}^{22}=\lambda_{R}^{13} / 36=\lambda_{R}^{12} / 36$, so that we obtain $C_{10 \mu \mu}=10^{-3}$ and $C_{10 e e}=-1.3$, which are able to explain the flavor anomalies. We are also taking $M_{\Phi_{4}}=M_{\phi_{4}^{5 / 3}}=M_{\phi_{4}^{2 / 3}}$ since the mass splitting cannot be large.

(iii) $\boldsymbol{\Phi}_{3} \& \boldsymbol{\Phi}_{4}$ scenario: In Sec. IV A we discussed the scenario where both fields, $\Phi_{3}$ and $\Phi_{4}$, contribute to the flavor anomalies and the connection between the predictions for $\mathcal{R}_{K}$ and $(g-2)_{\mu}$. In this case, the bound from $\mu \rightarrow e \gamma$ can be satisfied because the coupling of $\Phi_{4}$ to electrons is suppressed and the $\Phi_{3}$

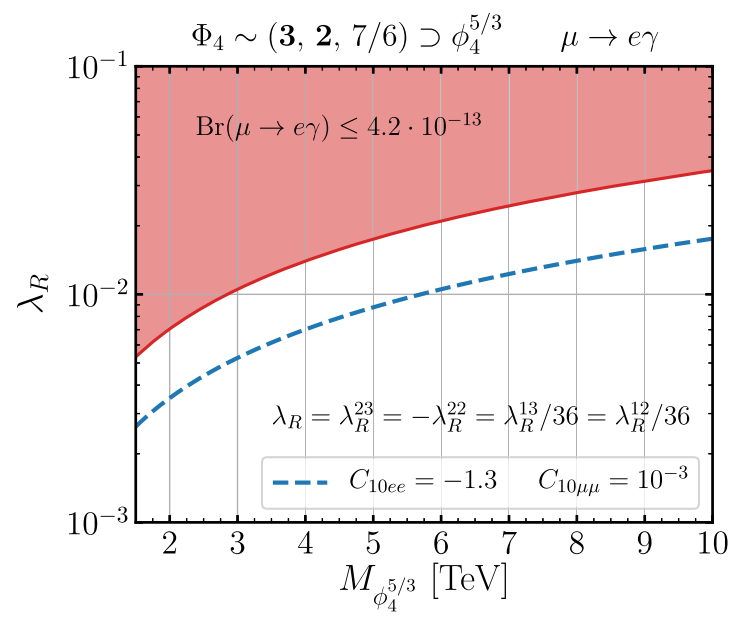

FIG. 10. Parameter space in the $\lambda_{R}$ vs $M_{\phi_{4}^{5 / 3}}$ plane, the region shaded in red is excluded by the experimental bound $\operatorname{Br}(\mu \rightarrow$ $e \gamma) \leq 4.2 \times 10^{-13}$ [77]. The other couplings have been fixed to $\lambda_{R}=\lambda_{R}^{23}=-\lambda_{R}^{22}=\lambda_{R}^{13} / 36=\lambda_{R}^{12} / 36$ so that we obtain $C_{10 \mu \mu}=$ $10^{-3}$ and $C_{10 e e}=-1.3$ which are able to explain the flavor anomalies.

contribution to such processes is also suppressed, and therefore, this bound can be neglected as in the first case discussed above.
[1] J. C. Pati and A. Salam, Lepton number as the fourth color, Phys. Rev. D 10, 275 (1974); Erratum, Phys. Rev. D 11, 703 (1975).

[2] P. Minkowski, $\mu \rightarrow e \gamma$ at a rate of one out of $10^{9}$ muon decays?, Phys. Lett. 67B, 421 (1977).

[3] T. Yanagida, Horizontal gauge symmetry and masses of neutrinos, Conf. Proc. C 7902131, 95 (1979).

[4] M. Gell-Mann, P. Ramond, and R. Slansky, Complex spinors and unified theories, Conf. Proc. C 790927, 315 (1979).

[5] R. N. Mohapatra and G. Senjanovic, Neutrino Mass and Spontaneous Parity Nonconservation, Phys. Rev. Lett. 44, 912 (1980).

[6] P. Fileviez Perez and M. B. Wise, Low scale quark-lepton unification, Phys. Rev. D 88, 057703 (2013).

[7] R. N. Mohapatra, Mechanism for Understanding Small Neutrino Mass in Superstring Theories, Phys. Rev. Lett. 56, 561 (1986).

[8] R. N. Mohapatra and J. W. F. Valle, Neutrino mass and baryon number nonconservation in superstring models, Phys. Rev. D 34, 1642 (1986).

[9] I. Doršner, S. Fajfer, A. Greljo, J. F. Kamenik, and N. Košnik, Physics of leptoquarks in precision experiments and at particle colliders, Phys. Rep. 641, 1 (2016).
[10] R. Aaij et al. (LHCb Collaboration), Test of lepton universality in beauty-quark decays, arXiv:2103.11769.

[11] B. Gripaios, M. Nardecchia, and S. A. Renner, Composite leptoquarks and anomalies in $B$-meson decays, J. High Energy Phys. 05 (2015) 006.

[12] R. Alonso, B. Grinstein, and J. Martin Camalich, Lepton universality violation and lepton flavor conservation in $B$-meson decays, J. High Energy Phys. 10 (2015) 184.

[13] L. Calibbi, A. Crivellin, and T. Ota, Effective Field Theory Approach to $b \rightarrow s \ell \ell^{(')}, B \rightarrow K^{(*)} \nu \bar{\nu}$ and $B \rightarrow D^{(*)} \tau \nu$ with Third Generation Couplings, Phys. Rev. Lett. 115, 181801 (2015).

[14] H. Päs and E. Schumacher, Common origin of $R_{K}$ and neutrino masses, Phys. Rev. D 92, 114025 (2015).

[15] D. Bečirević, N. Košnik, O. Sumensari, and R. Zukanovich Funchal, Palatable leptoquark scenarios for lepton flavor violation in exclusive $b \rightarrow s \ell_{1} \ell_{2}$ modes, J. High Energy Phys. 11 (2016) 035.

[16] R. Barbieri, C. W. Murphy, and F. Senia, B-decay anomalies in a composite leptoquark model, Eur. Phys. J. C 77, 8 (2017).

[17] A. Crivellin, D. Müller, and T. Ota, Simultaneous explanation of $\mathrm{R}\left(\mathrm{D}^{(*)}\right)$ and $b \rightarrow s \mu^{+} \mu^{-}$: The last scalar leptoquarks standing, J. High Energy Phys. 09 (2017) 040. 
[18] B. Capdevila, A. Crivellin, S. Descotes-Genon, J. Matias, and J. Virto, Patterns of New Physics in $b \rightarrow s \ell^{+} \ell^{-}$ transitions in the light of recent data, J. High Energy Phys. 01 (2018) 093.

[19] G. D’Amico, M. Nardecchia, P. Panci, F. Sannino, A. Strumia, R. Torre, and A. Urbano, Flavour anomalies after the $R_{K^{*}}$ measurement, J. High Energy Phys. 09 (2017) 010.

[20] G. Hiller and I. Nisandzic, $R_{K}$ and $R_{K^{*}}$ beyond the standard model, Phys. Rev. D 96, 035003 (2017).

[21] D. Buttazzo, A. Greljo, G. Isidori, and D. Marzocca, B-physics anomalies: A guide to combined explanations, J. High Energy Phys. 11 (2017) 044.

[22] N. Assad, B. Fornal, and B. Grinstein, Baryon number and lepton universality violation in leptoquark and diquark models, Phys. Lett. B 777, 324 (2018).

[23] L. Di Luzio, A. Greljo, and M. Nardecchia, Gauge leptoquark as the origin of B-physics anomalies, Phys. Rev. D 96, 115011 (2017).

[24] L. Calibbi, A. Crivellin, and T. Li, Model of vector leptoquarks in view of the $B$-physics anomalies, Phys. Rev. D 98, 115002 (2018).

[25] T. Faber, M. Hudec, M. Malinský, P. Meinzinger, W. Porod, and F. Staub, A unified leptoquark model confronted with lepton non-universality in $B$-meson decays, Phys. Lett. B 787, 159 (2018).

[26] S. Balaji, R. Foot, and M. A. Schmidt, Chiral SU(4) explanation of the $b \rightarrow s$ anomalies, Phys. Rev. D 99, 015029 (2019).

[27] B. Fornal, S. A. Gadam, and B. Grinstein, Left-right SU(4) vector leptoquark model for flavor anomalies, Phys. Rev. D 99, 055025 (2019).

[28] O. Popov, M. A. Schmidt, and G. White, $R_{2}$ as a single leptoquark solution to $R_{D^{(*)}}$ and $R_{K^{(*)}}$, Phys. Rev. D 100, 035028 (2019).

[29] S. Balaji and M. A. Schmidt, Unified SU(4) theory for the $R_{D^{(*)}}$ and $R_{K^{(*)}}$ anomalies, Phys. Rev. D 101, 015026 (2020).

[30] A. Angelescu, D. Bečirević, D. A. Faroughy, F. Jaffredo, and O. Sumensari, On the single leptoquark solutions to the $B$-physics anomalies, arXiv:2103.12504.

[31] G. Hiller, D. Loose, and I. Nišandžić, Flavorful leptoquarks at the LHC and beyond: Spin 1, J. High Energy Phys. 06 (2021) 080.

[32] W. Altmannshofer and P. Stangl, New physics in rare B decays after Moriond 2021, arXiv:2103.13370.

[33] C. Cornella, D. A. Faroughy, J. Fuentes-Martín, G. Isidori, and M. Neubert, Reading the footprints of the B-meson flavor anomalies, arXiv:2103.16558.

[34] R. Fleischer, R. Jaarsma, and G. Tetlalmatzi-Xolocotzi, Mapping out the space for new physics with leptonic and semileptonic $B_{(c)}$ decays, Eur. Phys. J. C 81, 658 (2021).

[35] D. Lancierini, G. Isidori, P. Owen, and N. Serra, On the significance of new physics in $b \rightarrow s \ell^{+} \ell^{-}$decays, arXiv:2104.05631.

[36] B. Abi et al. (Muon g-2 Collaboration), Measurement of the Positive Muon Anomalous Magnetic Moment to 0.46 ppm, Phys. Rev. Lett. 126, 141801 (2021).
[37] G. W. Bennett et al. (Muon g-2 Collaboration), Final Report of the muon E821 anomalous magnetic moment measurement at BNL, Phys. Rev. D 73, 072003 (2006).

[38] S. Borsanyi et al., Leading hadronic contribution to the muon 2 magnetic moment from lattice QCD, Nature 593, 7857 (2021).

[39] A. Freitas, J. Lykken, S. Kell, and S. Westhoff, Testing the muon g-2 anomaly at the LHC, J. High Energy Phys. 05 (2014) 145; Erratum, J. High Energy Phys. 09 (2014) 155.

[40] W.-C. Chiu, C.-Q. Geng, and D. Huang, Correlation between muon $g-2$ and $\mu \rightarrow e \gamma$, Phys. Rev. D 91, 013006 (2015).

[41] L. Calibbi, R. Ziegler, and J. Zupan, Minimal models for dark matter and the muon g-2 anomaly, J. High Energy Phys. 07 (2018) 046.

[42] K. Kowalska, E. M. Sessolo, and Y. Yamamoto, Constraints on charmphilic solutions to the muon g-2 with leptoquarks, Phys. Rev. D 99, 055007 (2019).

[43] R. Mandal and A. Pich, Constraints on scalar leptoquarks from lepton and kaon physics, J. High Energy Phys. 12 (2019) 089.

[44] I. Doršner, S. Fajfer, and O. Sumensari, Muon $g-2$ and scalar leptoquark mixing, J. High Energy Phys. 06 (2020) 089.

[45] I. Bigaran and R. R. Volkas, Getting chirality right: Single scalar leptoquark solutions to the $(g-2)_{e, \mu}$ puzzle, Phys. Rev. D 102, 075037 (2020).

[46] A. Crivellin, D. Mueller, and F. Saturnino, Correlating $h \rightarrow$ $\mu^{+} \mu^{-}$to the Anomalous Magnetic Moment of the Muon Via Leptoquarks, Phys. Rev. Lett. 127, 021801 (2021).

[47] R. Capdevilla, D. Curtin, Y. Kahn, and G. Krnjaic, A nolose theorem for discovering the new physics of $(g-2)_{\mu}$ at muon colliders, arXiv:2101.10334.

[48] M. J. Baker, P. Cox, and R. R. Volkas, Radiative muon mass models and $(g-2)_{\mu}$, J. High Energy Phys. 05 (2021) 174.

[49] C.-W. Chiang and K. Yagyu, Radiative Seesaw mechanism for charged leptons, Phys. Rev. D 103, L111302 (2021).

[50] B. Zhu and X. Liu, Probing light dark matter with scalar mediator: Muon $(g-2)$ deviation, the proton radius puzzle, arXiv:2104.03238.

[51] M. A. Buen-Abad, J. Fan, M. Reece, and C. Sun, Challenges for an axion explanation of the muon $g-2$ measurement, arXiv:2104.03267.

[52] D. W. P. Amaral, D. G. Cerdeño, A. Cheek, and P. Foldenauer, Distinguishing $U(1)_{L_{\mu}-L_{\tau}}$ from $U(1)_{L_{\mu}}$ as a solution for $(g-2)_{\mu}$ with neutrinos, arXiv:2104.03297.

[53] Y. Bai and J. Berger, Muon $g-2$ in lepton portal dark matter, arXiv:2104.03301.

[54] P. Athron, C. Balázs, D. H. Jacob, W. Kotlarski, D. Stöckinger, and H. Stöckinger-Kim, New physics explanations of $a_{\mu}$ in light of the FNAL muon $g-2$ measurement, arXiv:2104.03691.

[55] A. D. Smirnov, The minimal quark-lepton symmetry model and the limit on Z-prime mass, Phys. Lett. B 346, 297 (1995).

[56] M. Beneke, C. Bobeth, and R. Szafron, Power-enhanced leading-logarithmic QED corrections to $B_{q} \rightarrow \mu^{+} \mu^{-}$, J. High Energy Phys. 10 (2019) 232. 
[57] C. Bobeth, M. Gorbahn, T. Hermann, M. Misiak, E. Stamou, and M. Steinhauser, $B_{s, d} \rightarrow l^{+} l^{-}$in the Standard Model with Reduced Theoretical Uncertainty, Phys. Rev. Lett. 112, 101801 (2014).

[58] R. Fleischer, R. Jaarsma, and G. Tetlalmatzi-Xolocotzi, In pursuit of new physics with $B_{s, d}^{0} \rightarrow \ell^{+} \ell^{-}$, J. High Energy Phys. 05 (2017) 156.

[59] J. A. Bailey et al., $B \rightarrow K l^{+} l^{-}$decay form factors from three-flavor lattice QCD, Phys. Rev. D 93, 025026 (2016).

[60] A. Bharucha, D. M. Straub, and R. Zwicky, $B \rightarrow V \ell^{+} \ell^{-}$in the Standard Model from light-cone sum rules, J. High Energy Phys. 08 (2016) 098.

[61] W. Altmannshofer, P. Ball, A. Bharucha, A. J. Buras, D. M. Straub, and M. Wick, Symmetries and asymmetries of $B \rightarrow$ $K^{*} \mu^{+} \mu^{-}$decays in the Standard Model and beyond, J. High Energy Phys. 01 (2009) 019.

[62] R. Aaij et al. (LHCb Collaboration), Measurement of the $B_{s}^{0} \rightarrow \mu^{+} \mu^{-}$Branching Fraction and Effective Lifetime and Search for $B^{0} \rightarrow \mu^{+} \mu^{-}$Decays, Phys. Rev. Lett. 118, 191801 (2017).

[63] T. Aaltonen et al. (CDF Collaboration), Search for the Decays $B_{s}^{0} \rightarrow e^{+} \mu^{-}$and $B_{s}^{0} \rightarrow e^{+} e^{-}$in CDF Run II, Phys. Rev. Lett. 102, 201801 (2009).

[64] R. Aaij et al. (LHCb Collaboration), Test of lepton universality with $B^{0} \rightarrow K^{* 0} \ell^{+} \ell^{-}$decays, J. High Energy Phys. 08 (2017) 055.

[65] D. Bečirević, S. Fajfer, and N. Košnik, Lepton flavor nonuniversality in $b \rightarrow s \ell^{+} \ell^{-}$processes, Phys. Rev. D 92, 014016 (2015).

[66] J. P. Lees et al. (BABAR Collaboration), Search for $B \rightarrow$ $K^{(*)} \nu \bar{\nu}$ and invisible quarkonium decays, Phys. Rev. D 87, 112005 (2013).
[67] W. Altmannshofer et al. (Belle-II Collaboration), The Belle II physics book, Prog. Theor. Exp. Phys. 2019, 123C01 (2019); Erratum, Prog. Theor. Exp. Phys. 2020, 029201 (2020).

[68] O. Lutz et al. (Belle Collaboration), Search for $B \rightarrow h^{(*)} \nu \bar{\nu}$ with the full Belle $\Upsilon(4 S)$ data sample, Phys. Rev. D 87, 111103 (2013).

[69] M. Bauer and M. Neubert, Minimal Leptoquark Explanation for the $R_{D^{(*)}}, R_{K}$, and $(g-2)_{\mu}$ Anomalies, Phys. Rev. Lett. 116, 141802 (2016).

[70] S. Saad, Combined explanations of $(g-2)_{\mu}, R_{D^{(*)}}, R_{K^{(*)}}$ anomalies in a two-loop radiative neutrino mass model, Phys. Rev. D 102, 015019 (2020).

[71] D. Huang, A. P. Morais, and R. Santos, Anomalies in $B$-meson decays and the muon $g-2$ from dark loops, Phys. Rev. D 102, 075009 (2020).

[72] K. S. Babu, P. S. B. Dev, S. Jana, and A. Thapa, Unified framework for $B$-anomalies, muon $g-2$ and neutrino masses, J. High Energy Phys. 03 (2021) 179.

[73] A. Greljo, P. Stangl, and A. E. Thomsen, A model of muon anomalies, arXiv:2104.05730.

[74] D. Marzocca and S. Trifinopoulos, A minimal explanation of flavour anomalies: b-meson decays, muon magnetic moment, and the Cabbibo angle, arXiv:2104.05730.

[75] L. Lavoura, General formulae for $f(1)->f(2)$ gamma, Eur. Phys. J. C 29, 191 (2003).

[76] R. Benbrik and C.-K. Chua, Lepton flavor violating $l \rightarrow l^{\prime} \gamma$ and $Z \rightarrow l \bar{l} \prime$ decays induced by scalar leptoquarks, Phys. Rev. D 78, 075025 (2008).

[77] A. M. Baldini et al. (MEG Collaboration), Search for the lepton flavour violating decay $\mu^{+} \rightarrow \mathrm{e}^{+} \gamma$ with the full dataset of the MEG experiment, Eur. Phys. J. C 76, 434 (2016). 